\title{
AN ASSESSMENT OF RADIATION DAMAGE MODELS AND METHODS
}

Roger E. Stoller and Louis K. Mansur

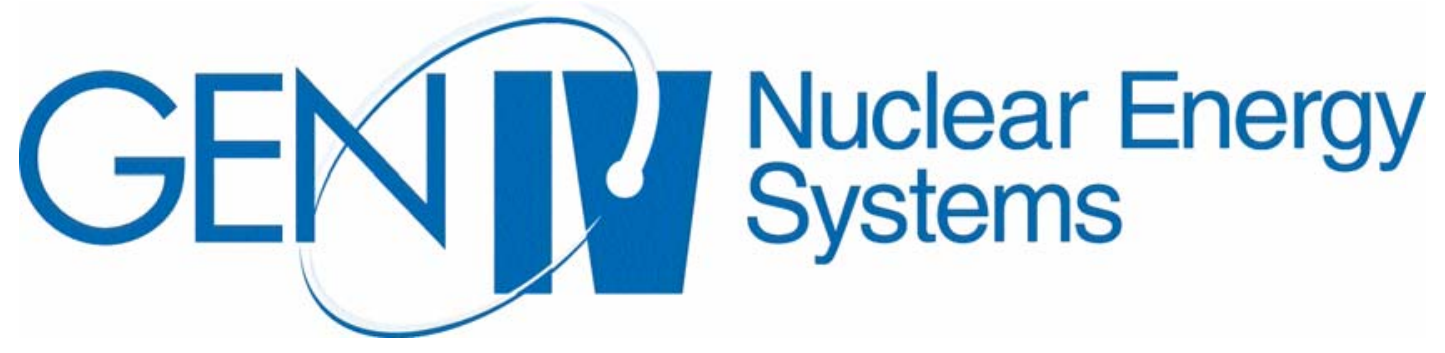

May 31, 2005 


\title{
AN ASSESSMENT OF RADIATION DAMAGE MODELS AND METHODS
}

\author{
Roger E. Stoller and Louis K. Mansur
}

May 31, 2005

Prepared for

Office of Nuclear Energy Science and Technology

Prepared by

OAK RIDGE NATIONAL LABORATORY

Oak Ridge, Tennessee 37831

managed by

UT-BATTELLE, LLC

for the

U.S. DEPARTMENT OF ENERGY

Under DOE Contract No. DE-AC05-00OR22725 


\section{TABLE OF CONTENTS}

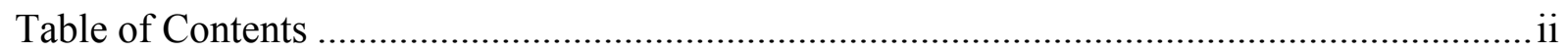

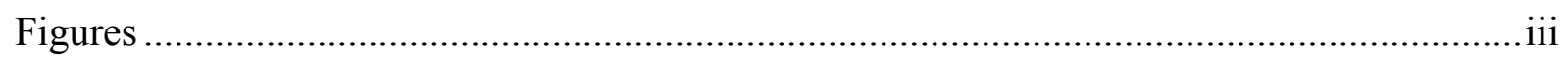

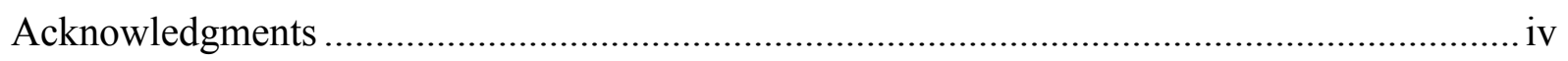

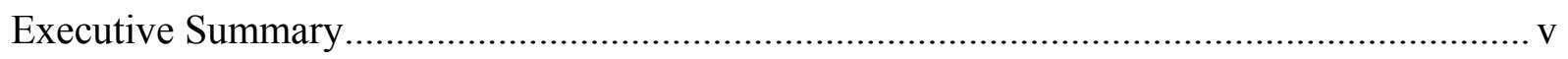

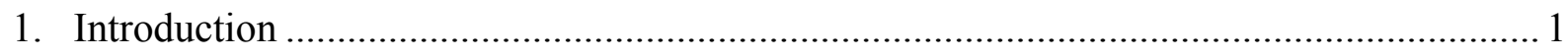

2. Radiation Environments and Materials for Generation IV Reactors ………...................... 2

3. Underlying Phenomena and Radiation-affected Properties ................................................. 2

4. Assessment of Computational Models and Methods: Current State of the Art and Needed Developments ...................................................................................... 10

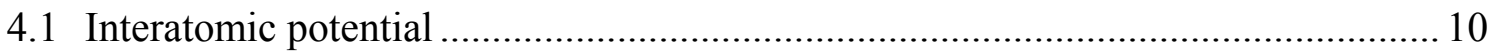

4.2 Molecular dynamics simulation ......................................................................... 13

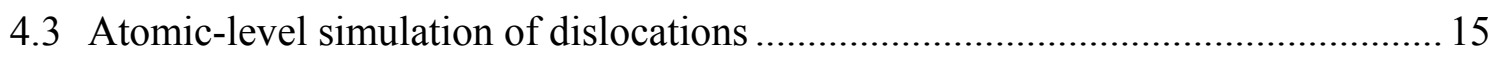

4.4 Kinetic Monte Carlo methods for defect transport and clustering............................ 17

4.5 Reaction rate theory of defect transport and clustering ………............................... 18

4.6 Dynamics of dislocation interactions with extended defects .................................... 24

5. Summary and Prioritization of Gen-IV Modeling Needs.................................................26

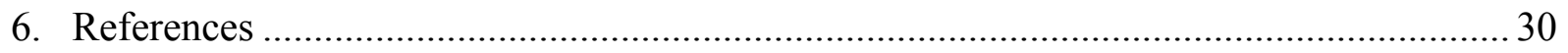




\section{FIGURES}

Figure 1. Characteristic time and energy scales for radiation effects in metallic alloys ......... 4

Figure 2. Schematic diagram of radiation damage processes in structural materials ............. 5

Figure 3. Volume change induced by void swelling in AISI 316 stainless steel irradiated to a fast fluence of $1.5 \times 10^{27} \mathrm{n} / \mathrm{m}^{2}$ at $533^{\circ} \mathrm{C}$.

Figure 4. Defect-free channels observed in niobium irradiated to a fast fluence of $4.4 \times 10^{22}$ $\mathrm{n} / \mathrm{m}^{2}(\mathrm{E}>1 \mathrm{MeV})$ at $50^{\circ} \mathrm{C}$ after straining to $6 \%$

Figure 5. Deformation map for Type 316 austenitic stainless steel at a plastic strain rate of $10^{-8} \mathrm{~s}^{-1}$. Unirradiated material is shown in (a), and material irradiated to $5 \mathrm{dpa}$ is shown for strain rates of $10^{-8} / \mathrm{s}$ and $10^{-10} / \mathrm{s}$ in (b) and (c), respectively [12]. See text for additional information......

Figure 6. Summary of computational tools and their uses in radiation damage modeling.... 11

Figure 7. Time dependence of point defect generation in MD cascade simulations in iron at $100 \mathrm{~K}$, note number of interstitials equals number of vacancies.

Figure 8. Stable configuration of vacancy (red spheres) and interstitial (green spheres) type defects from $20 \mathrm{keV}$ MD cascade simulations in iron at $600 \mathrm{~K}$, note large interstitial cluster.

Figure 9. Comparison of critical resolved shear stress $\left(\tau_{\mathrm{c}}\right)$ obtained from MS $(0 \mathrm{~K})$ and MD $(450 \mathrm{~K})$ simulations of an edge dislocation interacting with a $2 \mathrm{~nm}$ copper

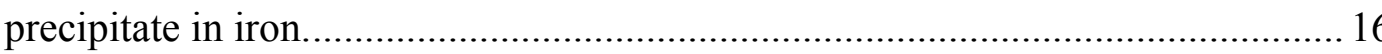

Figure 10. Comparison of fast reactor swelling data (a) and network dislocation density (b) with predictions of rate-theory-based model of microstructural evolution [46].... 21 


\section{Acknowledgments}

The authors acknowledge programmatic support from William Corwin and constructive review comments from Yury Osetskiy and Randy Nanstad.

This work is sponsored by the U.S. Department of Energy, Office of Nuclear Energy Science and Technology under contract DE-AC05-00OR22725 with Oak Ridge National Laboratory, managed by UT-Battelle, LLC. 


\section{Executive Summary}

The current state of development of the primary models used for investigating and simulating irradiation effects in structural alloys of interest to the U.S. DOE's Generation-IV reactor program are discussed. The underlying theory that supports model development is also described where appropriate. First, the key processes that underlie radiation-induced changes in material properties are summarized, and the types of radiation effects that subsequently arise are described. Future development work needed in order for theory, modeling, and computational materials science to support and add value to the Gen IV reactor materials program are then outlined. The expected specific outcomes and overall benefits of the required effort are: the knowledge to extrapolate material behavior to conditions for which there are no experimental data; systematic understanding of mechanisms and processes to enable confident interpolation between point-by-point experimental observations; acceleration of the development, selection, and qualification of materials for reactor service; and prediction of material response to real-world operating load histories which often involve a complicated superposition of time, temperature, radiation dose rate, and mechanical loading conditions.

Opportunities for international collaboration to accelerate progress in all of the required research areas are briefly discussed, particularly in the context of two well coordinated, broad-based research projects on modeling and simulation of radiation effects on materials that are currently funded in Europe. In addition to providing the opportunity for substantial leveraging of the DOE-funded activities in this area, these projects may serve as models for future development within the Gen-IV program. The larger of these two projects, which involves 12 European research laboratories and 16 universities, is called PERFECT and is funded by the European Union (http://www.fp6perfect.net). A smaller effort focusing on developing predictive models for fusion reactor materials is funded within the United Kingdom (http://users.ox.ac.uk/ roberts/sgrgroup/fusion/fusion_main.htm). Increased formal collaboration with these projects by DOE-funded materials scientists would be of substantial benefit to the Gen-IV program. 


\section{Introduction}

The performance of Generation IV (Gen-IV) reactors as a class will be determined largely by the behavior of advanced engineering materials. In particular, the adequacy and lifetime of materials utilized for the reactor cores and pressure vessels are crucial to the success of the Gen-IV reactor program. It is therefore essential to attain a highly informed initial selection of materials. In many cases, the operating conditions encountered by structural materials in GenIV reactors are much more challenging than those obtained with present day reactor technology. Many of the Gen-IV exposure conditions also lie outside the regimes investigated in previous research programs in radiation effects on materials. Thus, both new experimental data and predictions of expected behavior at conditions for which there are no experimental data will be required. In specific cases it is also likely that current materials available for reactor applications will not meet intended new service conditions and that development of improved materials, either by modification of existing alloys or design of new alloys, will be required to meet Gen-IV performance requirements. The most practical approach to achieving the required goals of informed material selection and quantitative predictions of material behavior is through an integrated program of theory and modeling in concert with appropriate experiments.

Fortunately, the theory and modeling of radiation effects on structural materials have been under development for many years. The motivation for these activities has been both the basic interest to understand material behavior in response to the new variable of radiation and the need to gain knowledge of the radiation response of materials for specific applications. These applications have included a wide range of fission reactors, fusion reactors and acceleratordriven spallation neutron sources. The modeling activities that are carried out under the GenIV program need to make every effort to leverage these previous and ongoing research activities.

The purpose of the present report is to assess the current state of development and describe the future work needed in order for theory, modeling, and computational materials science to support and add value to the Gen IV reactor program. Expectations for specific outcomes and overall benefits of this work are: the knowledge to extrapolate material behavior to conditions for which there are no experimental data; systematic understanding of mechanisms and processes to enable confident interpolation between point by point experimental observations; acceleration of materials development, selection, and qualification for reactor service; and prediction of materials response to real-world operating load histories which often involve a complicated superposition of time, temperature, radiation dose rate, and mechanical loading conditions.

First, the key processes that underlie radiation-induced changes in properties of materials are summarized. This section is written without necessarily constraining the description to follow the conceptual framework of models and methods that has been imposed on the phenomena to date. The types of radiation effects on properties are described. These include several forms of dimensional instability and progressive changes in mechanical behavior that can lead to 
losses of desirable structural properties and failures under load. All of these changes in properties are rooted in fine scale structure and composition, which are usually lumped under the terms microstructural and microcompositional evolution. Radiation effects on changes in the susceptibility of structural components to corrosion by the reactor coolant can also be important. However, the current state of development in modeling and the future work needed in this area are not analyzed in detail in the present report.

Theoretical methods that can be used for investigating and simulating radiation effects in materials on scales from the atomistic to macroscopic are described next. To greater or lesser degrees, variants of these methods have been applied to areas other than radiation effects in materials. Many of these activities have reached the stage of special sub-disciplines in materials science modeling and are employed by a number of specialized practitioners. Descriptions of each area are followed by an assessment of the current status and then by recommendations for and prioritizations of future work.

\section{Radiation Environments and Materials for Generation IV Reactors}

The radiation environments and key materials issues for the NGNP, SCWR, LFR and GFR as they relate specifically to theory and modeling are summarized in section 5 of Ref. [1]. More detailed descriptions of materials research and development needs and recommended activities for the SCWR, NGNP, and GFR are provided in Refs. [2] to [4], respectively. In a broader context, materials needs for Gen-IV reactors have been analyzed and compared to corresponding needs for fusion reactors and spallation neutron sources in Ref. [5], and the issue of materials modeling for fission and fusion reactors has been the subject of other reports $[6,7]$. The current report has drawn from all these resources in formulating the recommendations made in the final section.

In general, structural alloys in these reactor concepts will be required to perform to doses ranging from tens to one hundred dpa or more. The exception is the NGNP where, because of the neutron moderating effectiveness of the massive graphite structure in the core region, the displacement doses to most metallic components will be substantially lower than one dpa. Another common feature of the advanced reactor concepts is that the goal of higher thermal efficiency that leads to exposure of structural alloys within the reactor vessel to higher temperatures [8]. In normal operation these range from $500-620^{\circ} \mathrm{C}$ for the core components of the SCWR and a proposed low temperature version of the LFR, to $850^{\circ} \mathrm{C}$ for the GFR core barrel. Under off-normal conditions temperatures of certain components could rise to as much as $1200-1600^{\circ} \mathrm{C}$ for components in the GFR or NGNP.

\section{Underlying Phenomena and Radiation-affected Properties}

Irradiation of materials in reactor can lead to extensive changes in microstructure, microcomposition, and macroscopic properties. In the metallic alloys which are the primary focus here, the direct displacement of atoms is the primary driving force for radiation effects [9]. These atomic displacements are the result of elastic collisions between high-energy 
neutrons and the atoms in the material. Energy lost in other processes such as ionization and excitation do not contribute to atomic displacements in the energy range typical of fission reactor spectra. However, in materials such as the ceramics that may be used for some components in several of the Gen-IV concepts, electronic processes can cause substantial changes to properties. High energy neutrons $(\mathrm{E}>0.1 \mathrm{MeV})$ are primarily responsible for the displacement of atoms by elastic and inelastic collisions. Lower energy neutrons can displace atoms indirectly, for example, by causing $(n, \gamma)$ reactions where the neutron is absorbed and the nucleus emits a $\gamma$-ray. Conservation of momentum leads to the recoiling nucleus having enough energy to displace additional atoms. In addition to neutrons, high energy $\gamma$-rays in the reactor core can also cause indirect displacements. These $\gamma$-rays may create energetic electrons through Compton scattering and electron-positron pair production. When the $\gamma$-ray energy is above 1.5 to $2 \mathrm{MeV}$, the resulting electrons can have enough energy to displace atoms. However, the displacement cross-sections for high energy $\gamma$-rays are on the order of $10^{-3}$ those of fast neutrons, so that $\gamma$-ray displacements are usually negligible except under particular circumstances that apply to some water cooled and moderated reactors [10].

When an energetic neutron undergoes an elastic collision with an atom in the structural material, a so-called displacement cascade follows. This can be visualized as a series of elastic collisions which is initiated when the given atom recoils from the collision. This atom, which is called the primary knock-on atom (PKA), carries away a certain amount of kinetic energy which it dissipates in a sequence of collisions with other atoms. These are termed secondary knock-on atoms and they will in turn lose energy to third and subsequently higher ordered knock-ons until all of the energy initially imparted to the PKA has been dissipated. This process produces empty lattice sites (vacancies) and atoms left between lattice sites (interstitials). Fortunately, most vacancies and interstitials recombine, but those that do not are responsible for radiation-induced microstructural and mechanical property changes. The highest energy displacement cascades generated in iron-based alloys by fission reactor neutrons can create several hundred stable vacancies and interstitials. In addition, up to onehalf of these point defects cluster during the cascade cooling phase and these clusters directly seed the microstructure with nuclei that promote the formation of larger visible extended defects.

The continuous production and subsequent diffusion of point defects (and small point defect clusters) leads gradually to the formation of higher order point-defect clusters and other extended defects. The evolution of such extended defects in turn is responsible for the physical and mechanical property changes of the material. The left column of Figure 1 shows the characteristic time scales of the processes ranging from creation of point defects to alterations in macroscopic properties. The column on the right-hand side of Figure 1 shows the characteristic energy scales involved, ranging from the order of $1 \mathrm{MeV}$ for the initial neutron, down to the ordinary thermal energies for atomic diffusion. For iron atoms in steels, the energy of the displaced atom can be as high as approximately one-fifteenth the energy of the elastically scattered high energy neutron. This means that pka with kinetic energies of several tens of $\mathrm{keV}$ are common. Although the displacement events associated with a given pka are complete in about $10^{-11} \mathrm{~s}$, changes such as swelling, creep, and embrittlement 


\section{Time and Energy Scales for Radiation Effects from Displacement Damage}

\begin{tabular}{|c|c|}
\hline Time & Energy \\
\hline $\begin{array}{c}\text { Cascade creation } \\
10^{-15}-10^{-12} \mathrm{~s}\end{array}$ & $\begin{array}{c}\text { Neutron or Proton } \\
10^{5}-10^{7} \mathrm{eV}\end{array}$ \\
\hline Unstable matrix & Primary Knockon Atom \\
$10^{-11} \mathrm{~s}$ & $10^{3}-10^{5} \mathrm{eV}$ \\
\hline Interstitial diffusion & Displaced secondaries \\
$10^{-6} \mathrm{~s}$ & $1-1000 \mathrm{eV}$ \\
\hline Vacancy diffusion & Unstable matrix \\
$>0.1 \mathrm{~s}$ & $1 \mathrm{eV}$ \\
\hline Microstructural evolution & Thermal diffusion \\
$>10^{3} \mathrm{~s}$ & $\mathrm{kT}$ \\
\hline
\end{tabular}

Fig. 1. Characteristic time and energy scales for radiation effects in metallic alloys

generally can become significant at much longer times on the order of $10^{6} \mathrm{~s}$. Between the two regimes lie time scales of processes whereby the matrix recovers from the unstable condition during which some atoms may still have energies well above $k T$.

After these relatively rapid relaxations, the interstitials and vacancies diffuse by thermally activated processes from the region of the cascade. They may recombine with opposite type point defects created elsewhere; they may be absorbed at sinks such as pre-existing dislocations and grain boundaries; or they may cluster with like defects as well as minor alloying elements or impurities to create new extended defects. Because the recombination of vacancies and interstitials can prevent permanent radiation-induced changes, maximizing recombination is an important goal in seeking to make alloys resistant to radiation effects. Cavities, dislocation loops, and precipitates are important extended defects that are created by irradiation. Cavities, which are unstable at small sizes, can be stabilized effectively by the insoluble rare gas helium and/or hydrogen, both of which are unavoidably produced during neutron irradiation through $(\mathrm{n}, \alpha)$ and $(\mathrm{n}, \mathrm{p})$ transmutation reactions.

Besides the changes in microstructure, drastic changes in local composition can be produced. In an alloy, atoms of one type may preferentially exchange or couple with either type of diffusing point defect. Separation of the alloying constituents on a local scale can result from repeated associations of this type. For example, regions in the vicinity of a point-defect sink like a dislocation, grain boundary, or cavity may be significantly enriched or depleted in certain elements. This phenomenon is termed radiation-induced solute segregation.

Consequently, radiation-induced precipitation of a new phase having composition, structure 


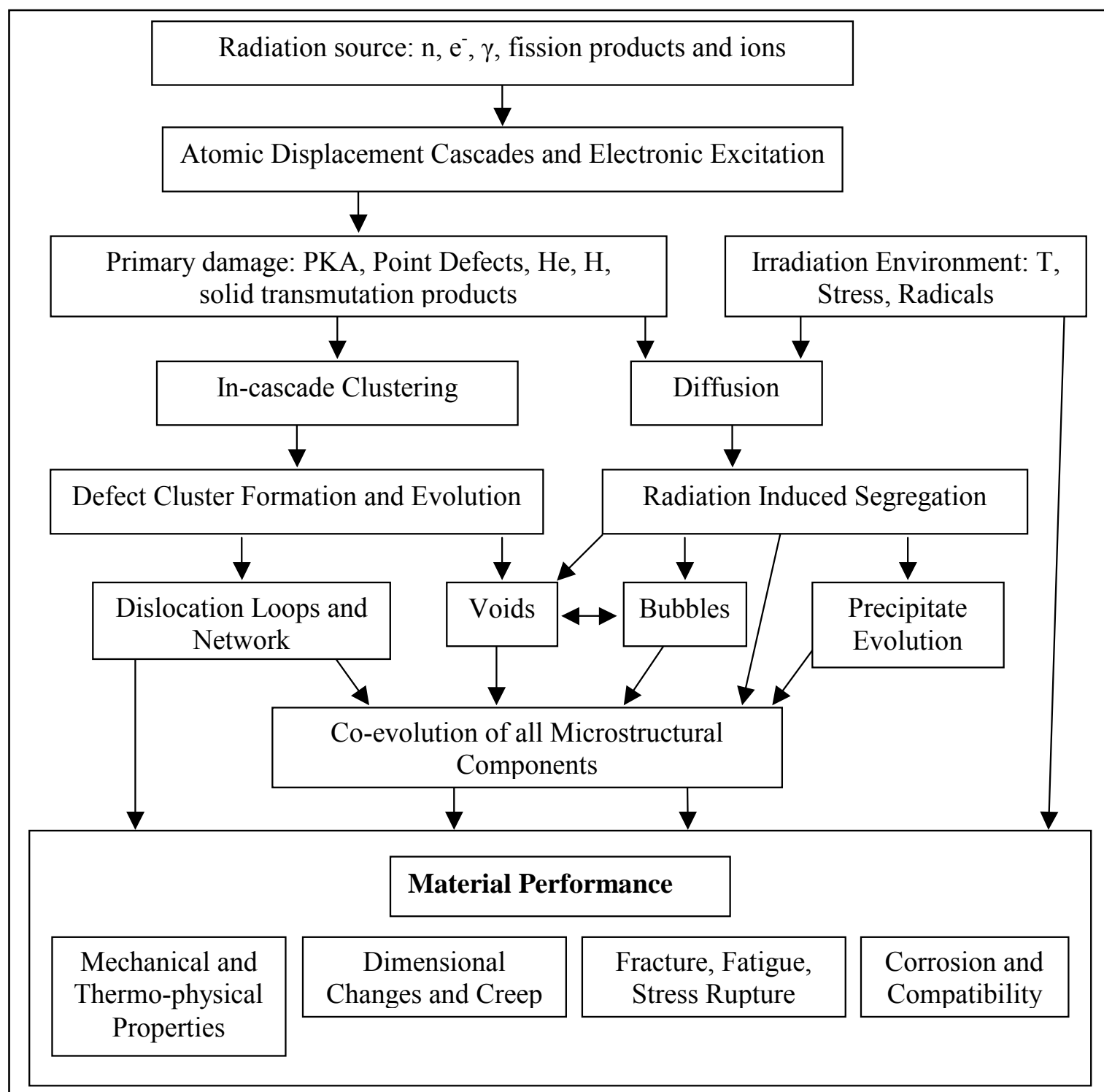

Fig. 2. Schematic diagram of radiation damage processes in structural materials

or properties different from that of the host matrix may occur, even though the average alloy composition would not lead to such a phase change under thermal conditions. Drastic changes in engineering properties of structural alloys can be produced as a result of these local microstructural and microcompositional changes. An overall schematic diagram of the relevant processes involved in radiation effects in structural materials is shown in Figure 2.

Macroscopic swelling of a material can occur when radiation-induced vacancies aggregate, leading to cavity formation and growth. This segregation of vacancies is possible because the corresponding interstitial atoms have been absorbed in net excess by dislocations. The preference or "bias" of dislocations for interstitial absorption is the result of the interaction between their long-range strain fields. Swelling is one of the most technologically significant radiation effects. Swelling levels of several tens of percent can result from high-dose 
irradiations of structural materials such as stainless steels. The dramatic change in specimen volume associated with void swelling is illustrated in Figure 3. The cavities responsible for swelling may be essentially empty voids, requiring only enough gas to stabilize them in their initial growth stages. However, cavities that are helium-filled bubbles can be formed on grain boundaries, and these grain boundary bubbles can significantly weaken the boundaries. The combination of matrix hardening by radiation-produced extended defects within the grain interiors and simultaneous weakening of grain boundaries by solute segregation and grain boundary bubbles may lead to embrittlement and intergranular fracture.

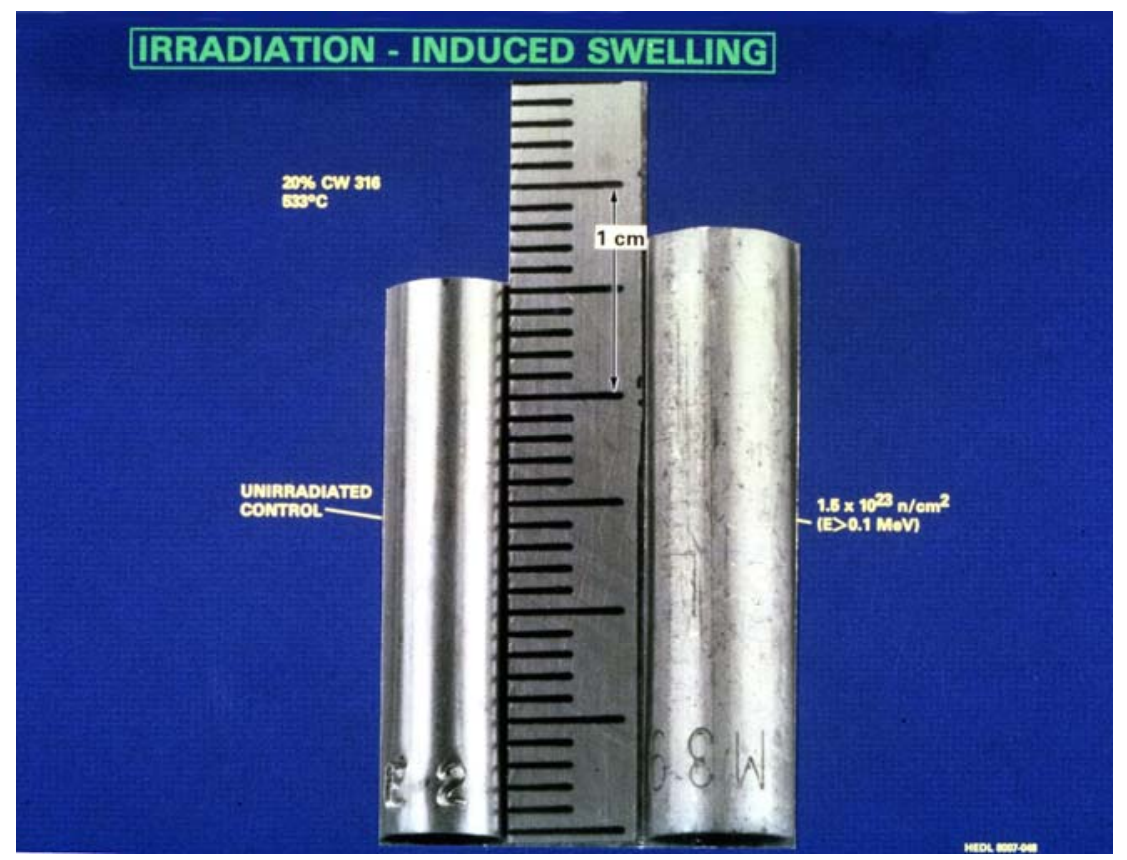

Fig. 3. Volume change induced by void swelling in AISI 316 stainless steel irradiated to a fast fluence $(\mathrm{E}>1.0 \mathrm{MeV})$ of $1.5 \times 10^{27} \mathrm{n} / \mathrm{m}^{2}$ at $533^{\circ} \mathrm{C}$ (F. A. Garner, PNNL, private communication).

Radiation-induced hardening is a property change associated with irradiation of almost all metals and alloys at low temperature (below $\sim 0.3$ of the melting temperature). The matrix hardening occurs due to the presence of radiation-induced defects which act as obstacles to dislocation motion. Based on simple geometric considerations of a dislocation traversing a slip plane which intersects randomly distributed obstacles with a given diameter and density, and using an appropriate approximation for the dislocation line tension, the increase in the critical resolved shear stress can be calculated using the well-known dispersed barrier hardening model $[11,12]$. The low-temperature hardening typically increases rapidly at low doses and transitions to a regime of apparent "saturation hardening" where the yield strength remains nearly constant or slowly increases as the dose is increased. Low-temperature hardening also leads to lower work hardening rates, as evidenced by a reduction in the ratio of the ultimate strength to the yield strength, and reduced uniform and total elongation. 
Flow localization is a phenomenon frequently associated with low temperature hardening [13]. This occurs when the typically uniform deformation microstructure observed in most unirradiated alloys is replaced by highly localized deformation after irradiation. Regions of very high deformation are surrounded by regions that have suffered little or no deformation. An example of such a microstructure is shown in Figure 4, where a TEM micrograph reveals the channels formed in a niobium specimen irradiated to a fast fluence of $4.4 \times 10^{22} \mathrm{n} / \mathrm{m}^{2}(\mathrm{E}>1$ $\mathrm{MeV}$ ) at $50^{\circ} \mathrm{C}$ after straining to $6 \%$ [14]. The phenomenon makes the material prone to failure along the channels or zones of high deformation. In certain alloys irradiated to high doses, the tensile ductility may be reduced from the tens of percent observed in unirradiated specimens to post-irradiation values ranging from tenths of a percent to values small enough to be below the detection capability of normal strain measuring equipment. In many cases, the evidence of flow localization is observed as a yield drop or region of strain softening in the stress-strain curve obtained in a standard tensile test.

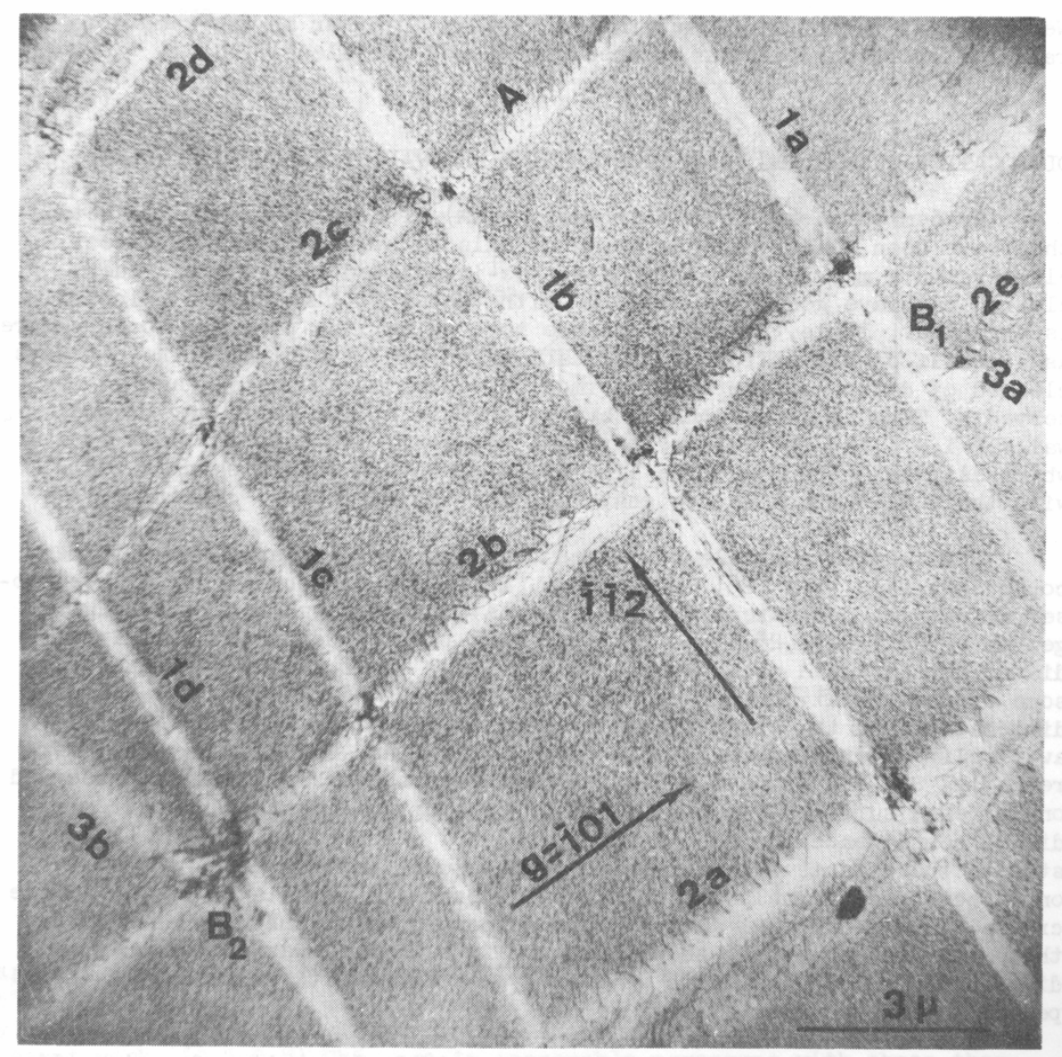

Fig. 4. Defect-free channels observed in niobium irradiated to a fast fluence of $4.4 \times 10^{22}$ $\mathrm{n} / \mathrm{m}^{2}(\mathrm{E}>1 \mathrm{MeV})$ at $50^{\circ} \mathrm{C}$ after straining to $6 \%[14]$.

Low temperature irradiation also reduces the fracture toughness of materials. This is typically observed as an increase in the ductile-to-brittle transition temperature (DBTT) in the case of bcc materials. The relationship between yield-stress increases and shifts to higher DBTT following irradiation is consistent with a simple model relating the DBTT (and DBTT shifts) to the strong temperature dependence of the yield strength and weak temperature dependence of the fracture stress [13]. Although the DBTT is normally determined through impact testing 
of notched specimens, and can be correlated with fracture toughness, the fracture toughness of the material is determined using specimens with sharp cracks that are more representative of the stress-strain gradients in a structural component. More recently, with developments in elastic-plastic fracture mechanics, the temperature dependence of fracture toughness of many iron-based bcc alloys has been shown to follow a universal relationship of fracture toughness vs. temperature when normalized to a specific specimen size. This universal curve has been termed the fracture toughness master curve [15] and has given the nuclear materials community the capability to characterize the fracture toughness of those materials with a relatively small number of relatively small specimens.

Irradiation creep generally occurs in materials that are stressed during irradiation. This volume-conserving, stress-directed shape change does not depend in an essential way on temperature. It can exceed the well-known thermally-activated creep by orders of magnitude. The mechanisms of irradiation creep are also more complex than thermal creep, for which values have been tabulated in materials handbooks as functions of stress and temperature for many engineering alloys. Irradiation creep is driven by processes that are similar to those responsible for swelling. A preferential partitioning of point defects again occurs.

Dislocations having different relative orientations to the stress axis absorb slightly different ratios of interstitials to vacancies. In addition, dislocations in groups absorb defects at different rates than do isolated dislocations. Thus, even though interstitials and vacancies are created by displacements in equal numbers, this asymmetric absorption can lead to climb of individual dislocations, which in turn results in creep. Creep caused by dislocation climb can result from either mass transfer between climbing dislocations oriented differently to the stress or by enabling the climbing dislocations to slip from pinning obstacles leading to a process termed climb-glide creep. Finally, transients in the behavior of the point defect concentrations can give rise to substantial irradiation creep, even at low temperatures [16].

Owing to the mechanisms and phenomena discussed above, the deformation modes of structural materials can be altered drastically by irradiation. These changes can be illustrated through the use of deformation maps that illustrate the operating regimes for which different mechanisms dominate the deformation behavior. An example of a two-dimensional stresstemperature plot is shown in Figure 5 for an austenitic stainless steel [12]. The primary effect of irradiation to $5 \mathrm{dpa}$ for a strain rate of $10^{-8} \mathrm{~s}^{-1}$ shown in a comparison of (a) and (b) is to increase the flow stress for dislocation glide. This causes twinning to be the dominant deformation mode up to room temperature, and the dislocation creep regime is extended to lower temperature compared to the unirradiated case A significant level of irradiation creep is predicted for the lower plastic strain rate of $10^{-10} \mathrm{~s}^{-1}$ shown in (c). Further information can be found in Ref. [12].

Alloys that are not designed to resist these effects of irradiation are generally inadequate for use as structural materials in fission reactors. Large-scale efforts have, therefore, been mounted to design alloys for irradiation performance. The principles applied to obtain improvements in alloy design have been largely discovered in basic studies of the physical mechanisms and kinetics of radiation effects [17]. Among the successes of this approach has 
(a)

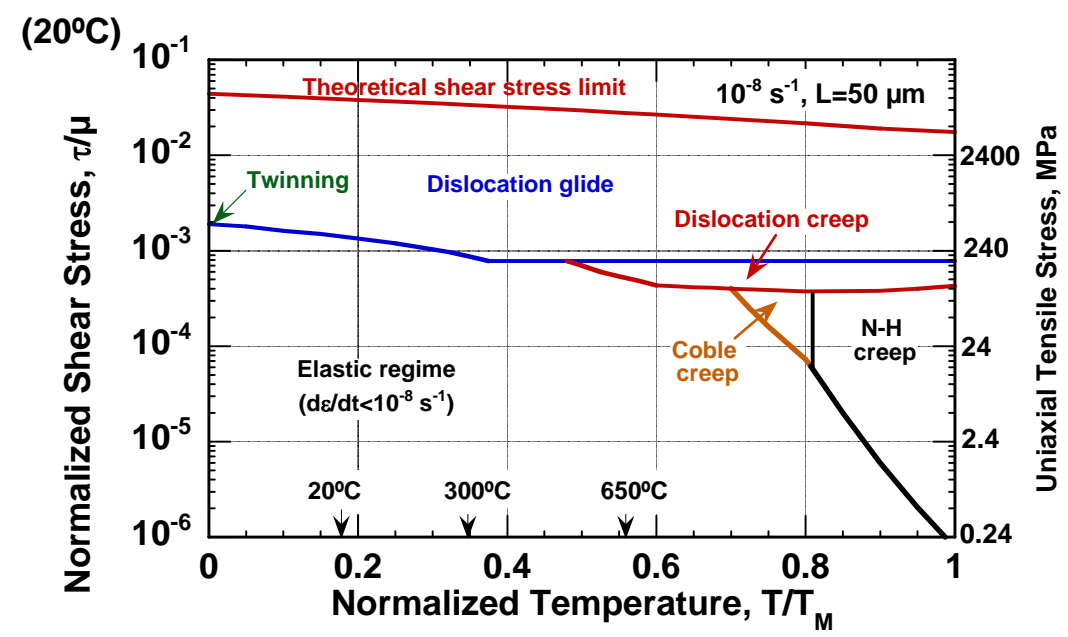

(b)

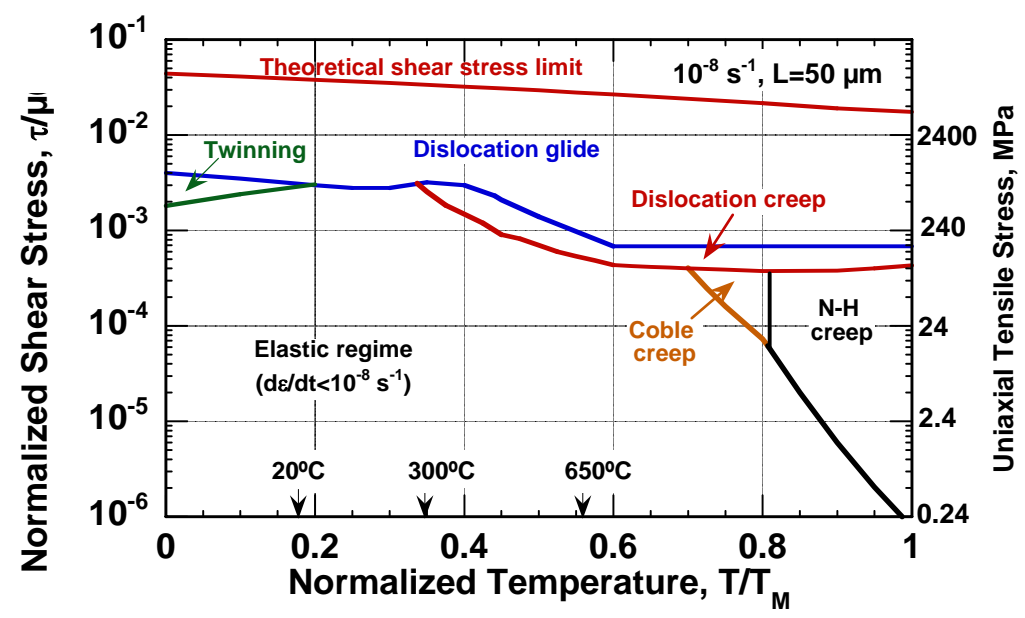

(c)

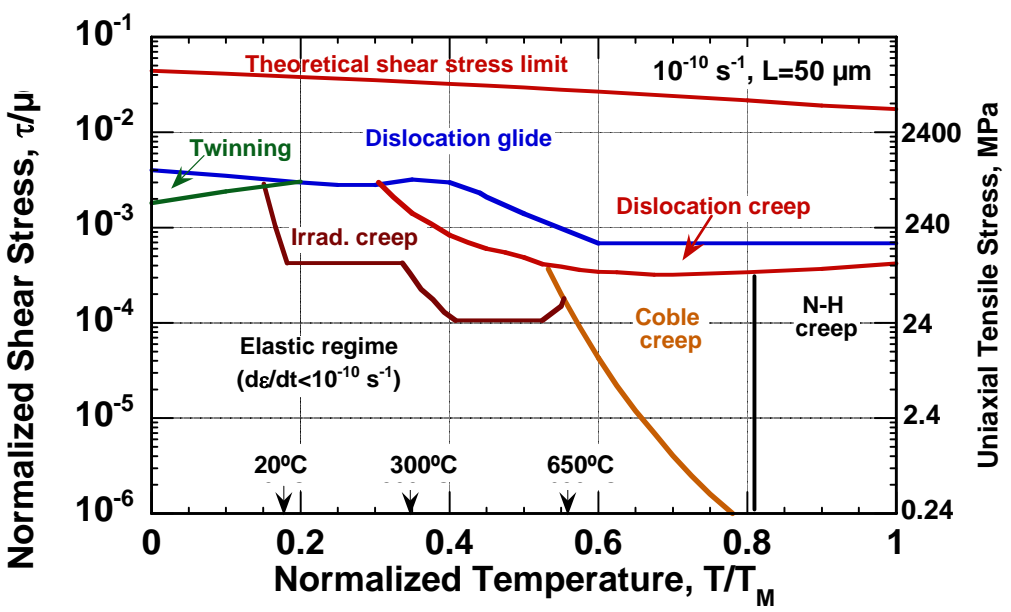

Fig. 5. Deformation map for Type 316 austenitic stainless steel at a plastic strain rate of $10^{-8} \mathrm{~s}^{-1}$. Unirradiated material is shown in (a), and material irradiated to $5 \mathrm{dpa}$ is shown for strain rates of $10^{-8} / \mathrm{s}$ and $10^{-10} / \mathrm{s}$ in (b) and (c), respectively [12]. See text for additional information. 
been the development of radiation-stable microstructures that feature a high density of small defect clusters and/or precipitates. Such microstructures lead to enhanced point defect recombination and trapping of transmutation-produced gases because their interface with the matrix provides an efficient trapping sites.

\section{Assessment of Computational Models and Methods: Current State of the Art and Needed Developments}

The theory and modeling tools required for use in research on Gen-IV reactor materials have been substantially developed under other programs and are under ongoing development by a larger group of experts active in many branches of solid-state physics and materials science. The smaller group of researchers active in the area of Gen-IV radiation damage simulation will continue to benefit by leveraging these other activities. In this section, some background information on the primary theory and modeling tools is presented and the current state of the art in their application is discussed.

The modeling tools can be grouped in four main classes that include different types of models as shown in Figure 6, along with their main output physical quantities. Atomistic methods such as $a b$ initio calculations, molecular dynamics (MD), and lattice Monte Carlo (MC) can be used to obtain detailed information about defect production and defect properties, while the mesoscopic and continuum models such as rate theory and object MC are used to simulate coarser scale microstructural evolution. These microstructural changes can then be used in micromechanics models to predict mechanical property changes.

\subsection{Interatomic potentials}

$\mathrm{MD}$ and some MC simulations require the use of an interatomic potential that represents the energy and forces associated with a configuration of atoms in a real material. To be usable for complex geometries and/or statistical averages, the potential needs to be computed rapidly. Many interatomic potentials have been developed and are reported in the literature, but none are necessarily adequate for simulation of the wide range of properties required for reactor materials. Simple pair potentials were the first to be developed, with only pair-wise interactions some physical parameters can not be reproduced. Many-body potentials (used here as a generic term covering glue, Finnis-Sinclair, embedded atom, modified embedded atom and effective medium theory potentials) are more robust and can reproduce a broad range of properties by appropriate fitting techniques when the necessary experimental values are available. These types of potentials have been the "state of the art" for twenty years. Lattice MC simulations can also be carried out without a full interatomic potential if appropriate interaction energies between the different atomic species that are present can be defined on the atomic lattice.

Historically, there has been an insufficient experimental database for robust parameterization and verification of potentials. The recent increased level of effort in developing interatomic potentials is partially based on the ability of ab initio calculations to provide this missing data, with modern teraflop machines providing the computational horsepower to enable the 


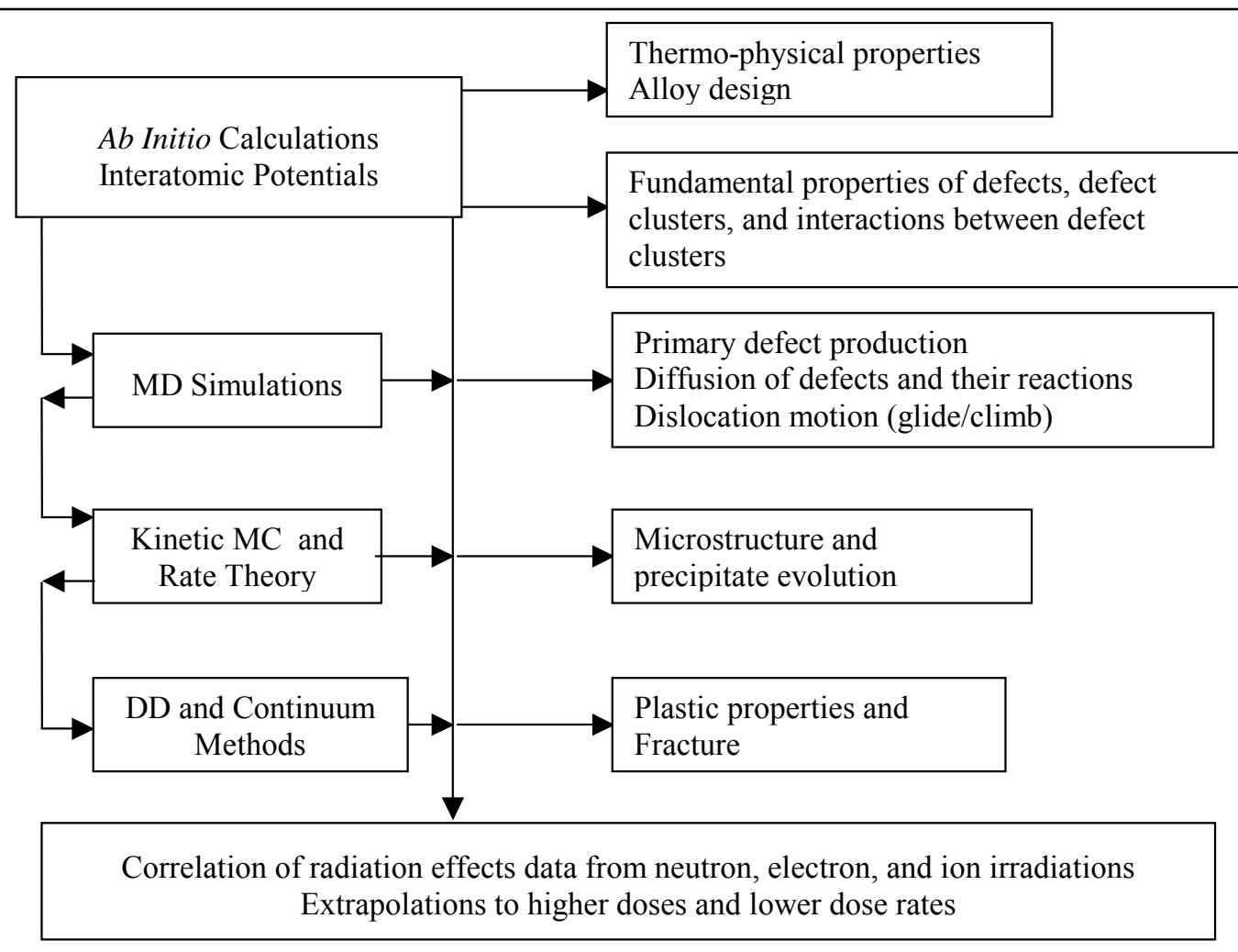

Fig. 6. Summary of computational tools and their uses in radiation damage modeling

required calculations. Where tested against new data, existing potentials have often proved disappointing. In the case of iron, which is the primary component in most relevant engineering alloys, problems include incorrect self-interstitial configurations and energies, and the absence of a satisfactory description of the austenite to ferrite phase transition. Some problems can be traced to parameterization of the potentials and may addressed by a reparameterization that is based on a more complete information base. Others, such as the absence of a physically sensible treatment of magnetization, point to more fundamental problems in the many-body potential concept.

Most effort for metallic phases has focused on elemental materials. These potentials are probably adequate for phenomena such as primary radiation damage formation in which most of the interesting events are controlled by atoms whose kinetic energy is much greater than kT. However, alloy potentials are required for simulation of many critical material properties. Potentials for some multi-component systems have been developed, but their predictive capability is typically disappointing. There are two challenges in the development of potentials for metal alloy systems. First, there is generally much less data available, though this can be rectified through the large-scale use of $a b$ initio methods. Second, the appropriate functional forms are not as well developed. Most potentials are based on simple pictures of bonding. In alloy systems, the nature of the bonding is inherently more complex, suggesting that more sophisticated potentials are needed. The ability to account for non-metallic impurities, such as carbon and phosphorus, and transmutant gases helium and hydrogen is 
even more problematic.

Recent progress in $a b$ initio calculations has allowed more accurate verification procedures to be applied to existing models, and highlighted some serious flaws. The prospects for rectifying these are good; some are simply a matter of reparameterization and extension to systems where insufficient underpinning data existed previously [18,19]; others require implementation of new models already proposed [20]; some may require the development of new approaches. The primary resource requirement for these developments is manpower, with stronger interactions between various research groups to attack the problems in a cohesive way. Recent progress in using ab initio results in the development of relevant new potentials includes $\mathrm{Fe}-\mathrm{Cr}$ [21] and $\mathrm{Fe}-\mathrm{He}$ potentials [22].

Validation and reparameterization of existing many-body models.

The technology for validation based on $a b$ initio simulation is mature. A database of structures and migration paths for point defects with and without impurities needs to be created. This will also benefit calculations using kinetic Monte Carlo (KMC) models. Efficient fitting techniques based on genetic algorithms can be employed to assist parameterization. Crucial $a b$ initio data not available currently includes those required to describe point defect migration and clustering mechanisms, dislocations core states and defect-dislocation interactions. Although necessary theory is generally available, the calculation of many of the required parameters in complex alloys is beyond the scope of current computing resources.

\section{Development of better models for magnetism and alloying.}

Recent developments suggest that it may be possible to create potentials with more complex functional forms than are currently used, while not unduly increasing the computational complexity. This is particularly important for the ferrite-based alloys that are of highest interest to many Gen-IV applications. Two new types have been proposed in Europe and could be exploited by the program. The two-band model represents the electronic structure on each atom by a single, local variational parameter $\mu$. This has been applied to describe the volume-collapse transition in Cs where $\mu$ represents s-d electron transfer [20]. The model has potential applicability to ferritic steels where $\mu$ would be the magnetization, and to materials where f electron localization is important. The functional complexity does not result in additional computational complexity thanks to the Hellmann-Feynman theorem, which means that the force can be evaluated without differentiating $\mu$.

Composition-dependent potentials offer the possibility of incorporating the desired alloy effects by making the potential dependent on the global as well as local electron density. This addresses such anomalies as the multiple bcc-fcc-hcp phase transitions observed with many short-range potentials under pressure. Here the functional form throughout the simulation is modified according to the composition and density of the MD simulation cell. This is practical since almost all MD simulations conserve particle number. There are some problems with constant pressure MD, since a fictitious term appears in the pressure - however since all 
applications of constant pressure MD already have a fictitious mass in the Lagrangian this issue should be tractable with perturbative corrections akin to the Pulay stresses encountered in plane-wave DFT. Simple composition-dependent potentials have been applied to dilute Fe$\mathrm{Cr}$ alloys which exhibit an oscillatory alloying energy that cannot be reproduced with conventional many-body potentials.

These two models offer tremendous promise for extending existing MD models to the complex multi-component steels and to properly understanding the atomic basis of the role of minority additives in determining bulk properties. This in turn opens the possibility of guiding development of materials, reducing the number of samples required to be synthesized for experimental testing. The underlying challenge is to develop a computationally convenient representation of the energy and forces between atoms that can be computed from ab initio methods. This can be viewed as an applied mathematics problem of finding a reliable representation of a known complex function.

\subsection{Molecular dynamics simulations}

Molecular dynamics (MD) is the most widely used method for atomic-scale simulation. For example, much of our current understanding of primary damage formation in displacement cascades [23-26], the behavior of point defects and their clusters [27,28], dislocation core properties, dislocation-defect interactions [29], and crack-tip processes, comes from MD simulation. In case of primary damage formation, recent advances in computing have permitted a substantial database of cascade simulations in iron to be developed in systems containing up to several million atoms. The dependence of point defect formation on parameters such as cascade energy and temperature have been described in detail using a Finnis-Sinclair potential [25], and comparisons between different potentials have been made [30]. Typical results from these simulations are shown in Figures 7 and 8 . The build-up to a peak number of displacements by about 0.5 to 1 ps shown in Figure 7 is followed by substantial recombination of vacancies and interstitials until a stable defect configuration is reached after 5 to 10 ps. This stable defect configuration is shown in Figure 8 for $20 \mathrm{keV}$ cascade at $600 \mathrm{~K}$, in which both isolated and clustered point defects can be observed.

In $\mathrm{MD}$, the classical equations of motion are integrated to obtain dynamical evolution of a system of atoms. Accurate integration requires time steps in the femtosecond range, limiting the total simulation time to less than a microsecond on today's processors. Direct MD is a powerful probe, giving the classically exact dynamical evolution of a system for a given interatomic potential. It provides for determination and prediction of material properties at the atomic scale and it offers a benchmark for the verification of higher-level models for materials evolution (kinetic MC, 3D dislocation dynamics, phase field models, rate theory, etc.) that involve assumptions and approximations about atomistic properties. Advances in interatomic potentials described above suggest that MD simulations may ultimately be compared directly with experimental results as both the techniques and computational resources improve. 


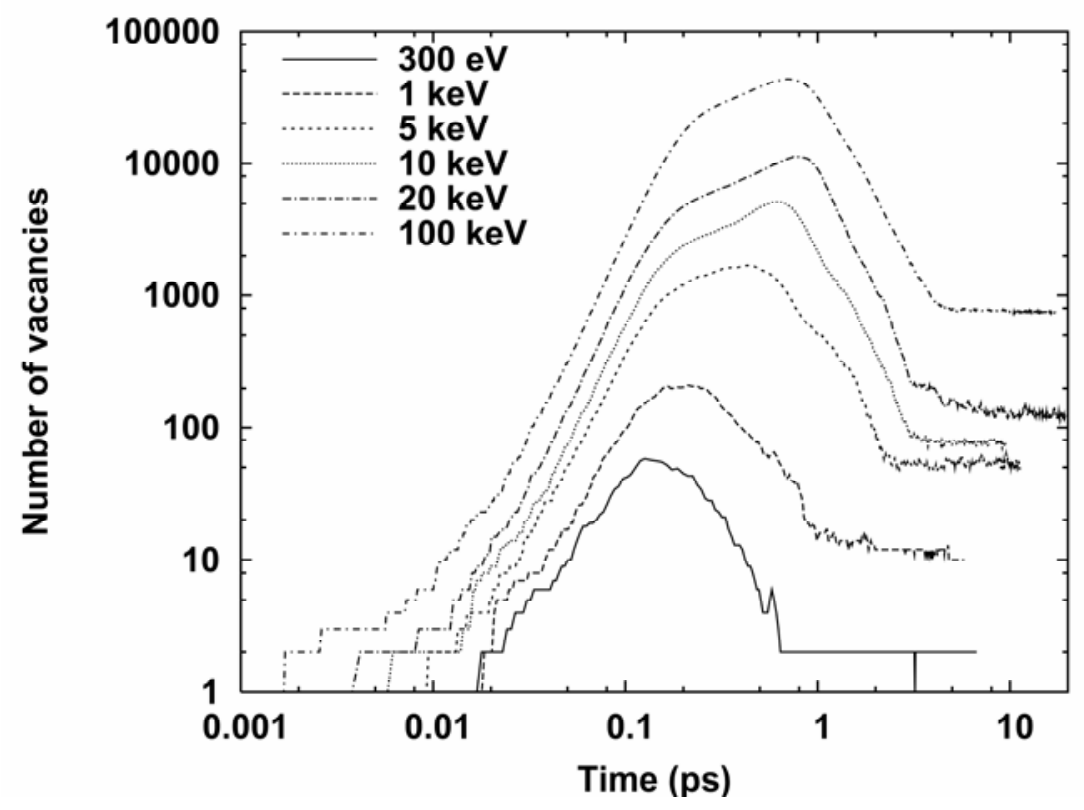

Fig. 7. Time dependence of point defect generation in MD cascade simulations in iron at $100 \mathrm{~K}$, note number of interstitials equals number of vacancies.

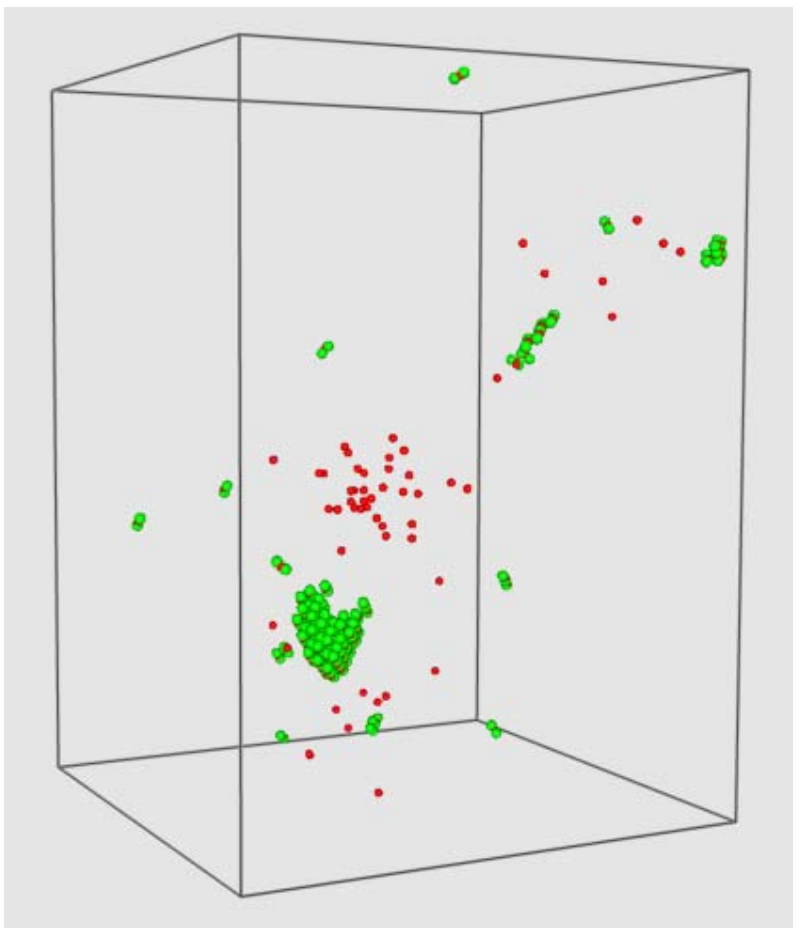

Fig. 8. Stable configuration of vacancy (red/dark spheres) and interstitial (green/light spheres) type defects from $20 \mathrm{keV}$ MD cascade simulations in iron at $600 \mathrm{~K}$, note large interstitial cluster.

Computer memory sets the upper limit for the number of particles, i.e. the system length scale. Fortunately, the static or dynamic properties of relatively small subsystems often determine the macroscopic materials behavior. This explains the widespread use and success 
in materials science of electronic band structure calculations, molecular statics and MD simulations. Moreover, MD parallelizes well via spatial decomposition, offering an easy path to extend the length scale. Simulations of a million atoms are routine, billion-atom simulations have been performed, and it should be possible to simulate $\sim 10^{11}$ atoms (= onemicron cube of $\mathrm{Fe}$ ) for approximately a microsecond on the next-generation leadership-class machine. Increasing model size will allow unprecedented direct examination of phenomena, such as multi-dislocation interactions and fracture. This means, for instance, that displacement cascades created by primary recoils with energy up to a few $100 \mathrm{keV}$ can be simulated, although electronic effects (excitation and electron-phonon coupling) are likely to remain difficult to incorporate in MD models. Since subcascade formation is common in most metals at the higher energies in this range, the use of Binary Collision Approximation (BCA) codes, tuned to MD results at overlapping energy, can also be expected to provide useful insight [31].

Time scales accessible to MD are more problematic. The calculation of the energy and interatomic force is by far the most expensive part of an MD simulation, so complexity in the interatomic potentials should be avoided. Model size is currently limited to about a thousand atoms if accurate quantum mechanically derived forces are used and larger systems mentioned in the preceding paragraph have to be described by more approximate force fields. Total simulation time and/or model size is expected to continue to increase proportionally to single-processor speed, but physical limitations in processor design will cause this to flatten out in the next 5-10 years. Consequently, direct MD simulations of systems with millions of atoms may never reach one millisecond, even with simple potentials. As many important activated phenomena (diffusion, defect clustering, void growth, grain growth, dislocation motion, creep, etc.) are active on time scales orders of magnitude beyond this, this poses a critical problem. Thus, methods of speeding up MD simulation are required. Although some techniques for accelerating MD have been developed [20], they are not applicable to many relevant problems. Depending on the nature of the problem being solved, the longer timescales may be investigated through the use of MC methods, kinetic (rate theory or phase field) models, or continuum methods.

\subsection{Atomic-level simulation of dislocations}

Computer modeling of dislocation processes relevant to damage microstructures can readily simulate several millions of atoms. With suitably designed periodic boundary conditions, dislocation glide over distances many tens of nm can be studied, and interaction with obstacles of realistic spacing can be treated. The importance of understanding dislocationobstacle interaction at the atomic level was demonstrated by Rodney and Martin [29] for the glide of an edge dislocation through an interstitial dislocation loop in $\mathrm{Ni}$. The absorption of the loop and jog formation, leading to pinning effects on the dislocation, was shown in detail. Since then, simulations of dislocation interactions with SFTs, loops, voids and precipitates have been reported, e.g. [32-34], including the screw dislocation [35]. With suitable boundary conditions for application of force or displacement, it is possible to extract quantitative information on the relationships between applied stress, strain rate, temperature, obstacle size 
and spacing for a given material $[32,34]$. However, with limited MD timescales $(\sim \mathrm{ns})$, plastic strain rates of at least $10^{6} \mathrm{~s}^{-1}$ are unavoidable. Thus, results on applied stress and/or dislocation configuration have to be tested against changes in strain rate and temperature in order to assess their possible applicability to much longer timescales.

As an alternative to MD, molecular statics (MS) may be employed to mimic the equilibrium state at $0 \mathrm{~K}$ by minimizing the potential energy of the system. With many atoms and complex reactions, it is not always possible to guarantee that a true minimum is achieved, and processes that depend on temperature may be missed. Nevertheless, by increasing the applied strain incrementally, equilibrium line and obstacle configuration under increasing stress can be found, e.g. $[32,34]$. This approach has the advantage that the results should be directly comparable with those given by models based on elasticity theory for the equilibrium of dislocations under stress. In fact, MS simulation of strengthening due to voids and precipitates in $\mathrm{Fe}[32,34]$ show that the earlier continuum treatments of these obstacles $[32,36]$ have wider applicability than expected.

The remaining challenge is to determine the extent to which information and mechanisms revealed in the atomistic simulations can be extracted and incorporated into dislocation dynamics (DD) simulations. In principle, this should produce nanoscale accuracy in the essentially-continuum DD models. However, some effects are difficult, if not impossible to account for using elasticity models. For example, Figure 9 illustrates the effect of temperature on the critical resolved shear stress for an edge dislocation to overcome a $2 \mathrm{~nm}$ (339 atom) copper precipitate in iron. The simulation involved two million iron atoms and a strain rate of $5 \times 10^{6} \mathrm{~s}^{-1}$. The values obtained in MS (0K) and MD (450K) simulations are printed in the

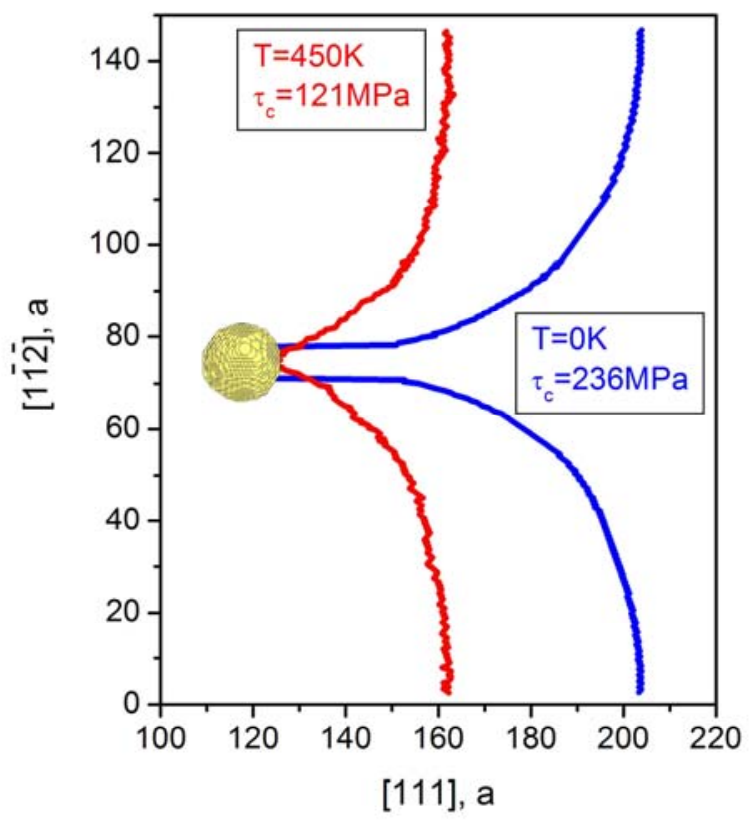

Fig. 9 Comparison of critical resolved shear stress $\left(\tau_{\mathrm{c}}\right)$ obtained from MS $(0 \mathrm{~K})$ and MD $(450 \mathrm{~K})$ simulations of an edge dislocation interacting with a $2 \mathrm{~nm}$ copper precipitate in iron (Yu. N. Osetskiy, private communication). 
figure which shows the critical dislocation configuration just before breaking free. A substantial level of effort in carrying out MD simulations for a variety of defect types is required to fully characterize the dependence of the critical resolved shear stress on temperature, strain rate, obstacle size and density, dislocation density, and obstacledislocation geometry.

\subsection{Kinetic Monte Carlo methods for defect transport and clustering}

A first step in the modeling of microstructure evolution is to predict the thermodynamic properties of complex systems, not always available experimentally. In addition to first principles calculations of cohesive energies, from which formation enthalpies [37] are deduced, very powerful numerical calorimetry methods based on static MC methods have been developed which give access to the thermodynamic functions of complex systems where the configuration entropy cannot be calculated explicitly [38]. This is the case of amorphous or strongly distorted crystalline structures.

The rigid lattice $\mathrm{KMC}_{-}$methods are extremely efficient for studying the incubation and nucleation of precipitates, and can now be validated with experiments at the same scale as the modeling box $\left(10^{-5} \mu \mathrm{m}^{3}\right)$ using $3 \mathrm{D}$ atom probe field ion microscopy (APFIM), a technique which has recently achieved close to atomic resolution. This combination of modeling and experimental observation can be used to identify the complex sequence of metastable and stable precipitation (or "dynamic phase diagrams") in alloys, to control materials strength and brittleness by influencing nucleation and coarsening, notably by identifying additives to enhance nucleation and hinder coarsening. They are particularly well adapted to test and integrate refined energetic and diffusion models, e.g. [39]. Recently, they have been upgraded to tackle irradiation effects by implementing all the interstitial diffusion mechanisms, point defect sources, and sinks. This permits calculating the point defect fluxes towards sinks which are responsible for radiation-induced segregation and precipitation (RIS \& RIP). As they give access to the details of evolution at the atomic scale for all the atoms in the calculation box, these techniques are demanding in CPU time (roughly proportional to the number of jumps performed) and are therefore limited to relatively small time and space scales, which in turn depend on temperature and atomic binding energies that control the jump probabilities. Also, they require knowledge of elementary parameters (binding and saddle point energies), which must be very accurate in view of the high sensitivity of kinetics to some of these parameters, whose number, in addition, becomes quite large in even moderately complex alloys. The massive input of first principles data is therefore crucial.

A limitation of the rigid lattice approach is that it does not allow for systems and phenomena with large size effects (i.e. large elastic constraints), at least not with an accuracy equivalent to that achieved for the effects of purely chemical bonds. For such situations, however, Relaxed lattice $\mathrm{MC}$ techniques are being developed to account for diffusion and precipitation in systems with large atomic and/or particle size effects, notably the coherent-incoherent transition in precipitation. These developments are promising, but are hindered by the enormous CPU time requirement [40]. 
Systems of larger dimension $\left(\mu \mathrm{m}^{3}\right)$ and comprising complex objects can be explored with the help of event-based KMC models, in which the MC algorithm is not applied to individual atomic jumps but to the probability of impingement or dissociation of populations of mobile defects and immobile objects [41]. This is possible at the expense of detailed spatial localization, which is no longer at the atomic scale. Such models are well adapted to account for recovery of complex point defect and defect-solute cluster populations in pure metals and dilute alloys during and after irradiation [42], and especially the effect of microstructural features such as dislocations, grain boundaries, precipitates, free surfaces etc, which of course requires that their sink and source strengths are correctly implemented.

In order to deal with concentrated, multi-component, heterogeneous systems over wider time and space scales, self-consistent mean field diffusion models are used. They describe the diffusive and thermodynamic properties at the same level of approximation, accounting for all the correlations between atomic jumps, and for both the thermodynamic and kinetic coupling of fluxes. The latter is of paramount importance for materials under irradiation as it gives rise to RIS at grain boundaries in stainless steels [43], for example. Also, such models are designed to take advantage of existing macroscopic data bases (e.g. DICTRA) and enrich them with atomic scale-based calculations.

\subsection{Reaction rate theory of defect transport and clustering}

In order to describe the overall evolution of single defect and defect cluster populations without space or time limitation, one can apply the reaction rate theory or cluster dynamics [17]. This theory can be used in conjunction with TEM and 3D Atom Probe experiments to develop models that directly simulate the results of experiments on an observable scale. For example, such models have been applied to understand the mechanisms related to the formation of extended defects and solute clusters responsible for the hardening and embrittlement of steels under neutron irradiation [44]. Results from the atomistic methods discussed above are used to provide critical parameters for these mesoscopic kinetic models, and can also provide some validation of model predictions in limited cases. Because of its broad use in radiation damage modeling, this theory is discussed in some detail here.

\section{Diffusion-reaction rate theory defect transport and clustering}

The flow diagram in Figure 2 above lists key processes that must be accounted for in describing the reaction pathways leading from initial point-defect production to large-scale macroscopic property changes. It gives a food chain sketch of the main issues that must be accounted for in a theoretical description.

The diffusion-reaction rate theory is a kinetic approach based on an effective medium modeled as a continuum. Each infinitesimal point is assigned both defect generation and loss. In a volume-averaged sense, the effective medium generates and loses the same number of defects as the actual material with spatially discrete sinks and temporally discrete events. Voids, dislocation loops, and point-defect clusters are "smeared out" in the continuum. Initially, this approach was based on its intuitive appeal. Later the approach was proven more 
rigorously. This approach is developed in detail in Ref. [17], where the original developments and earlier work in the field are reviewed. Many radiation-induced property changes are caused by the formation and evolution of point-defect clusters.

\section{Clustering theory applied to swelling}

Clustering theory starts with the discrete master equation:

$$
\frac{\partial C_{j}}{\partial t}=\sum_{k} w(k ; j) C_{k}-\sum_{k} w(j ; k) C_{j}+G_{j}-L_{j}
$$

Here, $C_{j}$ is the concentration of clusters of type $\mathrm{j}$. The transition rate $\mathrm{w}(\mathrm{k} ; \mathrm{j})$ is the rate per unit concentration of cluster type $\mathrm{k}$ of transitions to a cluster of type $\mathrm{j}$. The summations denote all reactions leading to a cluster of type $\mathrm{j}$ and all reactions removing a cluster of type $\mathrm{j}$, respectively. The term $G_{j}$ is the rate at which clusters of type $j$ are formed directly. The term $\mathrm{L}_{\mathrm{j}}$ is the loss rate of clusters of type $\mathrm{j}$ such as by diffusion to sinks and by destruction in cascades. To solve these equations, knowledge of many mechanisms is required, including details of the diffusion of each species, interaction parameters, and point-defect generation characteristics.

A direct approach to solving Equation 1 is by computation without further mathematical reduction of the equations. At high doses, large cavities require hundreds of millions of equations. With further approximations explained in Ref. [17], however, Equation 1 can be reduced to

$$
\frac{\partial \mathrm{C}_{\mathrm{j}}(\mathrm{t})}{\partial \mathrm{t}}=\frac{\partial}{\partial \mathrm{j}}\left\{\mathrm{C}_{\mathrm{j}}(\mathrm{t})[\mathrm{w}(\mathrm{j} ; \mathrm{j}+1)-\mathrm{w}(\mathrm{j} ; \mathrm{j}-1)]\right\}+\frac{1}{2} \frac{\partial^{2}}{\partial^{2}}\left\{\mathrm{C}_{\mathrm{j}}(\mathrm{t})[\mathrm{w}(\mathrm{j} ; \mathrm{j}-1)+\mathrm{w}(\mathrm{j} ; \mathrm{j}+1)]\right\}
$$

The first term in Equation 2 describes a drift in cluster-size space. The second term describes diffusion in size space. The first term describes the fact that a large cluster will grow steadily; the second term accounts for the fact that two different clusters introduced at the same time and the same size may differ in size at a later time through randomness in absorption of point defects.

Cavity-nucleation theory and cavity-growth theory start with Equation (2), as discussed in Ref. [17], where both theories are developed in some detail. The simplest form of cavitygrowth theory assumes that there are only point defects and point-defect sinks such as dislocations, cavities, grain boundaries, and precipitates. Clusters (like cavities and dislocation loops) are given the dimension $\mathrm{j}$, indicating the number of contained point defects, or the dimension $r$, indicating the cluster radius.

The first term in curly braces in Equation (2) is used in growth theory. Rewriting in terms of $r_{\mathrm{c}}$ gives: 


$$
\frac{\partial N\left(r_{c}, t\right)}{\partial t}=\frac{\partial\left[\dot{r}_{c} N\left(r_{c}, t\right)\right]}{\partial \mathbf{r}_{c}}
$$

Where $N\left(r_{c}\right) d r_{c}$ replaces $C_{j}$ as the number density of clusters at sizes between $r_{c}$ and $r_{c}+d r_{c}$. The important term in describing the cluster growth rate is $r_{c}$, the time derivative of the cavity radius. The growth rate of a cavity of radius $r_{c}$ is determined by the net flux of vacancy volume per unit cavity area per unit time

$$
\frac{d r_{c}}{d t}=\frac{\Omega}{r_{c}}\left[Z_{v}^{c} D_{v} C_{v}-Z_{i}^{c} D_{i} C_{i}-Z_{v}^{c} D_{v} C_{v}^{e}\left(r_{C}\right)\right]
$$

Here, $\Omega$ is atomic volume, $r_{c}$ is cavity radius, the D's are diffusion coefficients, the $Z^{c}$ 's are cavity-capture efficiencies, the C's are point-defect concentrations, and $C^{e}\left(r_{c}\right)$ is the thermal concentration of point defects at a cavity of radius $r_{c}$. The subscripts $i$ and $v$ denote interstitials and vacancies, respectively.

The concentrations are obtained by solving the continuity equations

$$
\begin{gathered}
\nabla\left(D_{v} \nabla C_{v}+\frac{D_{v} C_{v}}{k T} \nabla U_{v}\right)+G_{v}-R C_{v} C_{j}-K_{v} C_{v}=\frac{\partial C_{v}}{\partial} \\
\text { and } \\
\nabla\left(D_{i} \nabla C_{i}+\frac{D_{i} C_{i}}{k T} \nabla U_{i}\right)+G_{i}-R C_{v} C_{i}-K_{i} C_{i}=\frac{\partial C_{i}}{\partial}
\end{gathered}
$$

$\mathrm{G}$ denotes vacancy or interstitial generation rate. $\mathrm{R}$ is the recombination coefficient given by $4 \pi r_{r}\left(D_{i}+D_{v}\right)$, where $r_{r}$ is the radius of the recombination volume. The remaining terms describe losses to sinks. These are modeled in two ways. The terms with spatial derivatives describe leakage and drift to a sink modeled as discrete. The symbol $U$ is interaction energy with the discrete sink, and the $\mathrm{K}_{\mathrm{v}, \mathrm{i}}$ is the reaction-rate constant that describes the loss rates per unit point-defect concentration to sinks of type $\mathrm{K}_{\mathrm{v}, \mathrm{i}}$ which are treated as uniformly distributed throughout the continuum. Of these sinks, there may be $\mathrm{n}$ types (e.g., dislocations, cavities, dislocation loops, and others) and $\mathrm{K}_{\mathrm{v}, \mathrm{i}}=\Sigma_{\mathrm{n}} \mathrm{K}_{v, i}^{n}$, where $\mathrm{K}_{v, i}^{n}$ is defined as the product of a sink strength $S_{v, i}^{n}$ and the appropriate diffusion coefficient $D_{v, i}$. The sink strength $S$ is an expression that includes sink concentration, geometric factors, and sink efficiencies. Expressions for $\mathrm{S}$ have been derived for cavities, dislocation lines, dislocation loops, precipitates, and grain boundaries.

Both radiation-induced generation and thermal generation of defects are included in $\mathrm{G}_{\mathrm{v}}$. For example, thermal generation of vacancies is contributed by cavities, dislocations, and small clusters formed directly in cascades. In general, the clustering and thermal stability of interstitial and vacancy clusters formed in cascades may ultimately affect all property changes that depend upon point-defect production.

The right sides of Equations (5) and (6) express changes in defect concentration with time. 
However, except at low temperatures, the relaxation times for changes in the concentrations are generally much shorter than characteristic times for changes in $\mathrm{K}_{\mathrm{i}}$ and $\mathrm{K}_{\mathrm{v}}$ by microstructural evolution. When the right sides of Equations (5) and (6) are set to zero (steady-state conditions) and when all sinks are treated according to continuum descriptions, these equations become algebraic equations that are easily solved for concentrations.

Theoretical models of microstructural evolution based on these concepts can be directly compared with experimental results. For example, void swelling and the related dislocation evolution in austenitic stainless steels was heavily investigated in the U.S. liquid metal breeder and fusion reactor programs. Figure 10 compares the predictions of void swelling and dislocation density from a comprehensive rate-theory based model [46] with data obtained under fast reactor irradiation conditions. The void swelling is shown as a function of dose in Figure 10a, and the temperature dependence of the network dislocation density is shown in
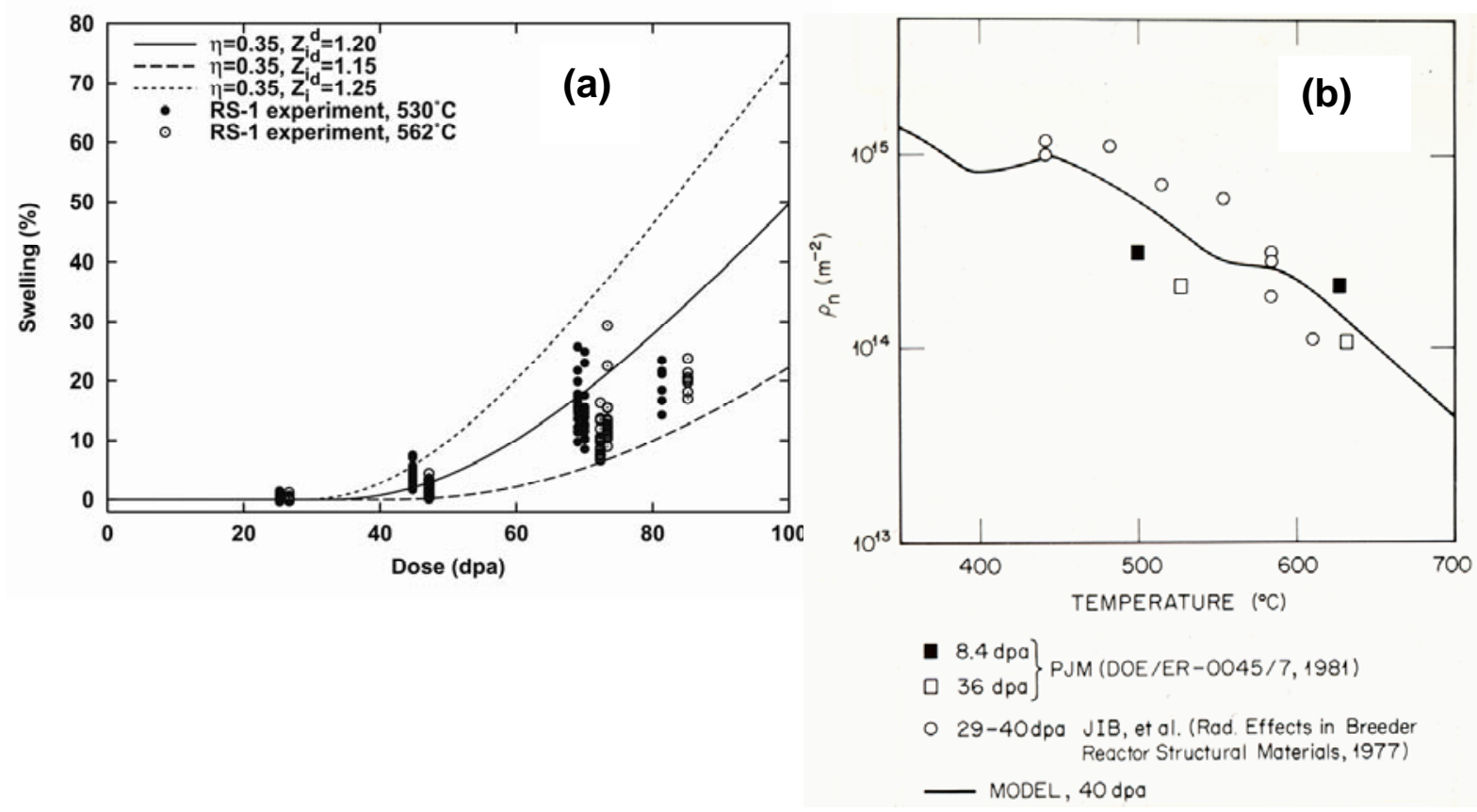

Fig. 10. Comparison of fast reactor swelling data (a) and network dislocation density (b) with predictions of rate-theory-based model of microstructural evolution [46].

10b. Along with such specific data comparisons, these models have also been used to elucidate important mechanisms in concert with special experiments. Three such example are discussed below.

\section{(a) Temperature Shifts}

Many irradiation experiments are conducted under conditions of accelerated damage rates in order to reach end-of-life exposure conditions in a reasonable time. The applicability of such data can be determined by careful modeling. By requiring invariance under different conditions in some parameters such as total defects absorbed at sinks or in net vacancies 
absorbed at cavities, powerful tools can be developed for predicting in shifts in irradiation variables [17]. For example, when one of the three irradiation parameters (dose rate, dose, or temperature) is changed, a shift in another of these variables may be prescribed that will maintain the same net flux of vacancies to cavities. Specifically, if the point-defect generation rate is changed from $G_{1}$ to $G_{2}$, then the temperature shift required for the same swelling rate under specific conditions is found as:

$$
T_{2}-T_{1}=\frac{\left[k T_{1}^{2} /\left(E_{v}^{m}+n E_{v}^{f}\right)\right] \ln \left(G_{2} / G_{1}\right)}{1-\left[k T_{1} /\left(E_{v}^{m}+n E_{v}^{f}\right)\right] \ln \left(G_{2} / G_{1}\right)}
$$

Here, $\mathrm{E}_{v}^{m}$ and $\mathrm{E}_{v}^{f}$ are the vacancy migration and formation energies and $\mathrm{n}=1$ or 2 depending on whether point-defect loss is dominated by sinks or by recombination. Equation (7) predicts a temperature shift of about $150^{\circ} \mathrm{C}$ for nickel or stainless steel in going from neutron irradiation $\left(\sim 10^{-6} \mathrm{dpa} / \mathrm{s}\right)$ to heavy ion irradiation $\left(\sim 10^{-3} \mathrm{dpa} / \mathrm{s}\right)$. The predictions of this temperature shift analysis are in good agreement with experiments and the analytical results were one of the clearest early successes of the theory.

\section{(b) Critical radius and critical number of gas atoms}

A very important concept in understanding how to control swelling is the cavity critical radius or, equivalently, critical number of gas atoms [45]. The existence of these critical quantities was uncovered by work with parts of the theoretical model presented above, which are reviewed in Ref. [17]. The concept of a critical number of gas atoms has explained numerous experimental observations and enabled the design of well-targeted experiments to probe swelling mechanisms.

When helium is generated by transmutation reactions or injected deliberately by ion accelerators, it is trapped in cavities and reduces thermal vacancy emission from cavity surfaces. When Equation 4 is plotted, it exhibits the following form. For a given number of contained gas atoms, $\mathrm{n}_{\mathrm{g}}$ there are two roots—one at a stable radius, $\mathrm{r}_{c}^{s}$, and one at a critical radius, $\mathrm{r}_{c}^{c}$. The cavity must achieve a size beyond $\mathrm{r}_{c}^{c}$ to grow by bias-driven growth. A point at a larger value than $\mathrm{r}_{c}^{c}$, denoted $\mathrm{r}_{c}^{o}$, is the critical radius with no gas. Gas makes achieving the critical radius easier. As more gas is added, $r_{c}^{c}$ is decreased and $r_{c}^{s}$ is increased up to a critical number of gas atoms, $\mathrm{n}_{\mathrm{g}}{ }^{*}$ where $\mathrm{r}_{c}^{s}$ and $\mathrm{r}_{c}^{c}$ meet at $\mathrm{r}_{c}{ }_{c}$. If any more gas is added, a critical radius no longer exists, and bias-driven cavity growth is inevitable. Thus, there are two qualitatively different paths to achieve bias-driven growth. The cavity may fluctuate about its stable size by stochastic events, on occasion exceeding $r_{c}^{c}$. Alternatively, the cavity may accumulate more than the critical number of gas atoms, whereby bias-driven growth begins with no fluctuations required.

The critical number of gas atoms has been useful in a variety of mechanistic studies and alloy design work. Three examples that can be cited are:

- The explanation of numerous observations of bimodal cavity size distributions.

- The understanding of the wide differences in swelling resistance of Fe-Ni-Cr alloys based on their specific nickel content. 
- The successful design of swelling-resistant alloys based on the formation of a fine precipitate distribution during irradiation.

\section{(c) Cavity growth rate}

In general, when Equation (4) is integrated and the evolving microstructural sink strengths are accounted for, swelling exhibits a sigmoidal shape versus displacement dose. It has been found that the ratio of dislocation strength to cavity-sink strength that appears in these equations (termed Q) is an important index to swelling regimes. Q is expressed as the ratio of the dislocation density to the sum of all cavity radii times another factor that includes the ratio of capture efficiencies and geometric constants. At low dose, $\mathrm{Q}>>1$ and the swelling per unit dose is greater than linear; for intermediate dose, $Q \sim 1$ and the swelling rate is linear; at high dose, $\mathrm{Q}<<1$ and the swelling rate is less than linear. Computations based on this analysis have been useful in understanding the relative swelling rates of different alloys in dose regimes for

which their swelling response dose may differ. In this analysis, these terms are not determined at absolute levels but by the relative evolution stage of micro-structure in the given alloy. The analysis is also useful in estimating the potential remaining for swelling in a given alloy. For example, if measurements show that $\mathrm{Q}<<1$ has already been reached, then it can be confidently predicted that the alloy will not be able to swell much more. Physically, this arises because all defects that are not annihilated by bulk recombination are annihilated in equal numbers at the dominant cavity sinks. Net partitioning of vacancies to cavities and interstitials to dislocations then cannot occur.

\section{Modeling Irradiation Creep}

Irradiation creep is an important process that has received much attention in theoretical modeling and in reactor and charged-particle irradiation experiments. A somewhat similar framework of theory and comparisons with experimental data has been developed for irradiation creep. Both steady-state or cumulative point-defect absorption mechanisms and transient mechanisms are important in irradiation creep. An example of the former is swelling-driven creep. Here, dislocations climb in response to the excess interstitial absorption that corresponds to the excess vacancy absorption at voids. However, in a stressed material, each increment of climb may lead to an increment of glide because dislocationpinning obstacles may be eventually climbed over.

Another type of mechanism that operates by cumulative point-defect absorption is stressinduced preferred absorption (SIPA). There are now several processes recognized under this heading by which dislocations whose burgers vectors are aligned with the stress axis will absorb slightly more radiation-produced interstitials than will nonaligned dislocations. The result is a gradual increase in the number of atom planes perpendicular to the stress axis and a decrease in the number parallel to the stress axis, resulting in creep deformation. In this sense, it is reminiscent of a type of Herring-Nabarro creep, which operates by an inverse mechanism (i.e., stress-induced preferred emission of thermal vacancies from dislocations whose burgers vectors are aligned with the stress axis). SIPA is a climb-only mechanism of irradiation creep. However, just as for swelling-driven creep, whenever a dislocation climbs there is the 
probability that it will climb over an obstacle and glide. Thus, in general, SIPA also induces climb-enabled glide creep. The latter process has been termed preferred absorption glide and, in fact, becomes larger than the climb-only SIPA process at high stresses.

An example of a transient mechanism is cascade-induced creep. Even though for cumulative processes like swelling, point-defect concentrations can be well approximated by the temporal and spatial averages, it is important to remember that in reality point-defect concentrations fluctuate wildly in time at any point because point defects are not created uniformly but in localized bursts. Particular dislocation segments then will climb back and forth in response to fluctuating vacancy and interstitial absorption. In some of these climb excursions in a stressed material, the dislocation may break away from the obstacle and glide away, thus giving rise to an increment of creep.

\subsection{Dynamics of dislocation interactions with extended defects}

The nature of dislocation motion within the microstructure of an irradiated material is a major factor to be considered for assessing the in-service integrity of reactor components. Motion can occur as a result of either applied stress, internal stress or chemical stress due to point defects, and gives rise to phenomena as varied as uniform flow, localized yielding, hardening, crack tip plasticity and creep. Until recently, most models of dislocation glide and climb in the presence of defects created by damage fluxes were based on the elasticity theory of dislocations, with line shape under stress estimated using the line tension approximation. This approximation neglects the effects of self-interaction between different parts of the line, which, for dislocations overcoming obstacles, can have an important influence on the flow stress. A dislocation with self-stress can be modeled as a flexible line constructed of piecewise segments. Although self-stress was first treated in this way over 30 years ago [47], it was only possible to consider a few hundred segments for a few tens of iterations. Ideally, 3D dislocation dynamics models of plasticity on the micro-meso scale require the simulation of 0.1-100 mm of line length (for a dislocation density of $10^{14} \mathrm{~m}^{-2}$ ), and thus contain typically $10^{5}-10^{8}$ segments. They should also be able to achieve a total plastic strain at realistic strain rates (e.g. $10 \%$ at $10^{-6} \mathrm{~s}^{-1}$ ). These aims cannot be met at the moment. Hence, new, more efficient techniques for computing the field of segments and their interactions with each other and damage microstructure are required.

In spite of current computational limits, dislocation dynamics simulations are becoming an increasingly important tool in the computational materials research [48,49]. Over the last several years there have been considerable advances both in the general DD methodology and in its implementation on massively parallel computers. The uniqueness of DD is its ability, on one hand, to incorporate the atomistic mechanisms of dislocation motion and interactions (among dislocations and with other elements of material microstructure) and, on the other hand, to directly compute the plastic strength of crystalline materials. Thus, DD provides a much-needed bridge between the length and time scales of the fundamental physical mechanisms and the length and time scales relevant for engineering applications.

Continuum-based DD models have uncertainties associated with singularities in the 
dislocation stress field and the loss of validity of the elasticity description at the atomic scale. These limitations do not apply to MD simulation of dislocation behavior. Hence, MD modeling can provide detailed information on obstacle forces [32] and dislocation core effects [50] for incorporation in larger scale DD simulation. However, the requirements on MD for treatment of plasticity at the length and time scales of interest are many orders of magnitude beyond current computing power. For example, limits on simulated time restrict the modeling of dislocation glide to strain rates of about $10^{6}$ per second, whereas rates a billion times smaller are of primary concern. For dislocation-controlled creep, the rates of interest are orders of magnitude smaller again. Thus, the limitations and benefits of using this method have to be recognized.

Three major challenges for further development of the DD approach are that (1) the unit mechanisms of dislocation motion and interaction are multiple and complex, (2) one needs to trace the evolution of very large groups of dislocations over extended periods of time while accessing the length and time scales relevant for microstructure evolution and its effects on yield, flow, strain hardening and strain localization, and (3) development of connections between DD and continuum plasticity theory. Recent achievements of $4-10 \%$ of plastic strain in a direct DD simulation suggest that the approach is on its way to becoming computationally efficient, through the use of massively parallel computing and more advanced numerical algorithms. The most serious of the remaining computability challenges is to scale DD simulations down to lower straining rates $\left(\sim 10^{-5}\right)$ and yet maintain their computational efficiency. The problem here is to be able to handle multiple time scales observed in the evolution of large dislocation populations, where periods of relative calm (slow motion of lines) are interspersed with bursts of very high activity over which small groups of lines moves very fast and experience multiple collisions.

Parallel DD implementations face their own problems. The first one is a programming challenge: the DD models deal with the ever-changing topology of dislocation lines and line networks requiring rather complex data structures and careful bookkeeping. On parallel machines, this challenge is dramatically amplified due to the need to handle the network topology across the boundaries of computational domains. The second challenge is a natural tendency of dislocation lines to cluster in space (owing to the long-range interactions among the lines) and develop highly heterogeneous distributions of degrees of freedom making it difficult to achieve a good load balance. The code ParaDiS recently written at LLNL has shown much promise in addressing these challenges [51].

DD simulations of radiation-damaged materials share the challenges of fidelity and computability and add others. As an example, the need to account for the atomistic details of dislocation interactions with obstacles such as precipitates, SFTs, dislocation loops, and a variety of other point defect (and defect-solute) clusters places even higher demands on the computational throughput of the DD codes. Still more complex and computationally challenging is the need to describe, within a single model, the concurrent evolution of dislocation-, alloy- and radiation defect-microstructures which is a key issue for predictive modeling of fission and fusion reactor materials. Several attempts have been made to couple 
KMC lattice simulations of the radiation defect microstructure with the DD simulations. While possible in principle, such coupling makes both DD and KMC simulations still more expensive than they are in isolation. At the same time, the phase field models that have been successfully used in the past for modeling alloy microstructure evolution were recently advanced to model dislocations and their motion. However, the phase-field DD models cannot compete with the line-tracking DD when it comes to combining high resolution with computational efficiency. As a possible way to reduce the computational complexity while achieving a degree of coupling between dislocation and defect sub-systems, it appears possible and in fact desirable to combine the line-tracking DD method (to handle the dislocation sub-structure) with a mesh-based phase-field approach (to handle the alloy field evolution).

\section{Summary and Prioritization of Gen-IV Modeling Needs}

The basis for much of the required Gen-IV reactor modeling activities has been laid in research done under other DOE and precursor agency programs; these include the earlier program to develop materials for liquid metal fast breeder reactors, the Fusion Materials Program, and radiation effects work funded by the Office of Basic Energy Sciences. Scientists involved in the broader field of computational materials science have also made substantial progress relevant research, particularly in the areas of $a b$ initio calculations and atomistic simulation techniques. Researchers funded by the Gen-IV (and AFCI) program must maintain close ties with those involved in these other programs in order to take maximum advantage of their results. This leveraging will both accelerate the pace of required research and reduce the cost to the program. At the same time, it is important to recognize that support for research on the effects of radiation on materials is being reduced elsewhere within the DOE and a more substantial effort will be required within the Gen-IV Program if materials research and development objectives are to be met.

In a generic sense, only modest extensions to existing models are needed for many aspects of Gen-IV materials research, but the specific needs of materials development for the Gen-IV reactor program suggest several near-term modeling objectives that should be pursued. The most challenging of these are related to the goal of employing higher temperatures for either higher thermal efficiency or to obtain higher coolant outlet temperatures for hydrogen production. Advanced ferritic-martensitic steels with good irradiation resistance have been developed in recent years, but the their use is limited to temperatures below about $550^{\circ} \mathrm{C}$. The best candidate material for the higher temperature applications is based on the same class of ferritic-martensitic steels, but obtains higher strength through oxide-dispersion strengthening (ODS). The best of these steels are strengthened by a high density of mixed-oxide particles with a diameter of only a few nanometers [52-54]. The thermal performance and a limited amount of irradiation data on these steels is encouraging but little modeling research has been carried out. Such work should be initiated in order gain the understanding that will permit a confident prediction the performance of the ODS steels under long-term irradiation at high temperatures. 
The complexity (primarily the number of constituent elements) of the ODS steels will limit the scale and range of atomistic simulations that can be carried out for this material. For example, this would preclude carrying out the $a b$ initio calculations necessary to develop a complete empirical alloy potential that could be applied in MD simulations. However, a substantial campaign of first principle calculations could be initiated to obtain the interaction energies between the various atomic species in sufficient detail to provide understanding of the underlying physics that is responsible of the behavior of the nanometer-sized oxide clusters that give these materials their desirable performance. These interaction energies could then be applied in lattice KMC simulations of solute behavior. The $a b$ initio results could be supplemented by the use of results from related research on iron-chromium alloys [55], and additional thermodynamic calculations using software such as Thermo-Calc and DICTRA. The KMC simulations could then be used to investigate the formation and evolution of the oxide clusters responsible for the high-temperature strength of the ODS steels. Both the thermo-mechanical and irradiation response of the oxide clusters need to be examined. Recent work directed at radiation effects in low-alloy reactor pressure vessel steels that follows this overall approach [56-58] provides some confidence about the level of progress that can be made.

These same atomic interaction energies can also be used to guide the development of a simplified interatomic potential that could be employed in MD simulations of dislocation defect interactions to examine possible strengthening mechanisms from the oxide nanoclusters. Although they appear to be structurally coherent with bcc lattice of the ferritic alloy host, the very large amount of strengthening they impart implies that the clusters must be nearly impenetrable obstacles. Based on some APFIM observations, the potential role of solute segregation pinning pre-existing dislocations needs to be investigated. This research may also be amenable to a combination of KMC and MD-based dislocation dynamics simulations.

In addition to the specific challenges associated with the ODS steels, further improvements in existing models for extended defect nucleation and growth are needed to account for alloying (largely thermodynamic) effects in many of the other candidate materials. Much of this research can be carried out in the rate theory framework described above, and in combination with phase field models of precipitate evolution [59,60]. Necessary, but unknown, material parameters can be obtained from $a b$ initio calculations, with increasing computational capability enabling solutions for more complex alloys. These more advanced kinetic models could then be used to predict the high-dose microstructural evolution of candidate materials under relevant Gen-IV reactor exposure conditions.

Further development and exploitation of both MD-based and 3D dislocation dynamics is required to understand the mechanisms leading to radiation-induced hardening, particularly at the lower exposure temperatures where high hardening can be associated with dislocation channeling and flow localization. Fine, radiation-induced defect structures can be destroyed during interactions with rapidly moving dislocations. The local softening that occurs leads to non-uniform deformation that is potentially catastrophic. Recent MD dislocation dynamics 
simulations have provided some insight into possible defect destruction mechanisms for small voids and stacking fault tetrahedra, but much more research is required to understand the behavior of the full complement of extended defects - including dislocation loops and second phase precipitates. Information on atomistic mechanisms can then be reduced to more simple rules that can be applied in $3 \mathrm{D}$ continuum dislocation dynamics models to predict flow behavior under realistic loading conditions.

Finally, it is important to link the microstructural predictions to changes in mechanical properties that ultimately limit material performance and material choices. The limiting property is primarily a function of the irradiation temperature, stress and stress state, and dose. At lower temperatures (on a homologous temperature scale) hardening-induced embrittlement may be dominant, dimensional instabilities such as swelling and the potential embrittling effects of radiation-induced solute segregation are more important at intermediate temperatures, and thermal creep and creep-rupture are prominent at high temperatures. Irradiation creep can be significant at low to intermediate temperatures and fatigue must be considered in components subject to cyclic loading.

As a result, the relationship between the microstructure and a range of local microconstitutive properties must be established. This will require the development of integrated multiscale models that combine the results of dislocation-obstacle interactions with true stress-strain constitutive and plasticity models that can be used in finite element codes. Integrated models will link applied stresses and strains to the micromechanical constitutive and fracture models through finite element simulations of the development of internal stress and strain field distributions. These fields drive local deformation and fracture processes. These models must provide the critical link between atomistic and/or microstructural processes and the continuum level loading conditions in order to account for size scales, geometry, loading conditions, and stochastic effects associated with inherently heterogeneous materials. On a relatively long timescale, continuum dislocation dynamics may provide a basis for direct simulations of deformation in complex alloy microstructures.

Advanced micromechanics models of the relation between the microstructure and local fracture properties must be developed. These will also require multiscale integration of submodels. For example, dislocation dynamics methods may be embedded in finite element models to account for the role of so-called trigger particles in the crack initiation and arrest, and the blunting of microcracks. KMC or phase field models may used to predict the growth and precipitation of coarse-scale brittle phases both in the matrix and at grain boundary phases. Under some Gen-IV irradiation conditions, sufficient levels of helium and hydrogen may be generated to increase the probability of intergranular fracture. The effect of both these gases and brittle grain boundary must be accounted for in new, more detailed fracture models.

Progress in all of the required research areas can be accelerated by taking greater advantage of opportunities for international collaboration. In particular, there are two examples of well coordinated, broad-based research projects on modeling and simulation of radiation effects on materials that are currently funded in Europe. These projects could serve as models for a 
larger DOE Gen-IV effort, while also providing a significant opportunity for collaboration and leveraging of research not funded by the U.S. DOE. The larger of these two projects, which involves 12 European research laboratories and 16 universities, is called PERFECT and is funded by the European Union (http://www.fp6perfect.net). A smaller, but still challenging research effort on developing predictive models for fusion reactor materials is funded within the United Kingdom by their Engineering and Physical Sciences Research Council (http://users.ox.ac.uk/ roberts/sgrgroup/fusion/fusion_main.htm). Some U.S. researchers who are partially funded by the Gen-IV project are informally collaborating with their European colleagues involved in the two efforts just mentioned. Specific funding to participate in these collaborations would be of substantial benefit to the Gen-IV program. 


\section{References}

1. R. E. Stoller and L.K. Mansur, "Modeling and Microstructural Analysis: Needs and Requirements for Generation IV Fission Reactors, ORNL/TM-2003/242, Oak Ridge National Laboratory, May 2004.

2. J. Buongiorno, et al., "Supercritical Water Reactor (SCWR) Survey of Materials Experience and R\&D Needs to Assess Viability," INEEL/EXT-03-00693 (Rev. 1), Idaho National Engineering and Environmental Laboratory, September 2003.

3. G. O. Hayner, et al., "Next Generation Nuclear Plant Materials Selection and Qualification Program Plan,” INEEL/EXT-03-001128 (Rev. 0), Idaho National Engineering and Environmental Laboratory, November 7, 2003.

4. W. R. Corwin, et al., "The Gas Fast Reactor (GFR) Survey of Materials Experience and R\&D Needs to Assess Viability," ORNL/TM-2004/99, Oak Ridge National Laboratory, April 30, 2004.

5. L. K. Mansur, et al., "Materials needs for fusion, Generation IV fission reactors and spallation neutron sources: similarities and differences,” J. Nucl. Mater. 329-333 (2004) 166-172.

6. R. E. Stoller, G. R. Odette and H. L. Heinisch, An Integrated Program of Theoretical, Experimental and Database Research for the Development of Advanced Fusion Materials, Whitepaper prepared for the US Department of Energy, Oak Ridge National Laboratory (1999)

7. Workshop on Advanced Computational Materials Science: Application to Fusion and Generation IV Fission Reactors, R. E. Stoller, S. J. Zinkle, J. A. Nichols, and W. R. Corwin (Eds), ORNL/TM-2004/132, Oak Ridge National Laboratory, June 2004.

8. Higher Temperature Reactor Materials Workshop, T. R. Allen, et al. (Eds), ANL-02/12, Argonne National Laboratory, August, 2002.

9. L. K. Mansur and K. Farrell, J. Nucl. Mater. 244 (1997) 212-218.

10. R. E. Stoller, "Primary Damage Formation in Irradiated Materials," JOM (formerly Journal of Metals) 48 (1996) 23-27.

11. A.K. Seeger, "On the Theory of Radiation Damage and Radiation Hardening," 2nd UN Conf. on Peaceful Uses of Atomic Energy vol. 6, United Nations, New York, 1958, pp. 250-273.

12. S. J. Zinkle and G. E. Lucas, "Deformation and Fracture Mechanisms in Irradiated fcc and bcc Metals," in Fusion Materials Semiannual Report for period ending June 302003 , DOE/ER-0313/34, Oak Ridge National Laboratory, 2003, pp. 101-125.

13. M. S. Wechsler, "Radiation Embrittlement of Matals and Alloys," Fundamental Aspects of Radiation Damage in Metals, Vol. II, CONF-751006-P2, M. T. Robinson and F. W. Young, Eds., U.S. ERDA, Washington, DC, 1975, pp. 991-1009. 
14. R.P. Tucker, S.M. Ohr and M.S. Wechsler, "Radiation Hardening and Transmission Electron Microscopy in Niobium," Radiation Damage in Reactor Materials, Vol. I, IAEA, Vienna, 1969, pp. 215-233.

15. ASTM E1921-05 Standard Test Method for Determination of Reference Temperature, $\mathrm{T}_{0}$, for Ferritic Steels in the Transition Range, ASTM International, West Conshohocken, PA.

16. R. E. Stoller, M. L. Grossbeck and L. K. Mansur, "A Theoretical Model of Accelerated Irradiation Creep At Low Temperatures by Transient Interstitial Absorption," Effects of Radiation on Materials, 15th International Symposium, ASTM STP 1125, R. E. Stoller, A. S. Kumar and D. S. Gelles, Eds., American Society of Testing and Materials, Philadelphia, 1992, pp. 517-529.

17. L. K. Mansur, "Mechanisms and Kinetics of Radiation Effects in Metals and Alloys," in Kinetics of Non-Homogeneous Processes, edited by G. R. Freeman, Wiley-Interscience, New York 1987, pp. 377-463.

18. G. J. Ackland, M. I. Mendelev, D. J. Srolovitz, S. Han and A. V. Barashev, J. Phys.: Cond. Mat. 16 (2004) S2629-S2642.

19. G.J. Ackland and S.K. Reed, Phys. Rev. B 67 (2003) 174108.

20. A.F. Voter, F. Montalenti and T.C. Germann, Annual Rev. Mater. Res. 32 (2002) 321.

21. J. Wallenius, I. A. Abrikosov, R. Chakarova, C. Lagerstedt, L. Malerba, P. Olsson, V. Pontikis, N. Sandberg, and D. Terentyev, J. Nucl. Mater. 329-333 (2004) 1175-1179.

22. T. Seletskaia, Yu. N. Osetsky, R. E. Stoller, and G. M. Stocks, Phys. Rev. Let. 94 (2005) 046403:1-4, and "Calculation of Helium Defect Clustering Properties in Iron using a Multi-scale Approach," presented at 2005 TMS Symposium on Microstructural Processes in Irradiated Materials, San Francisco, February, 2005, submitted to J. Nucl. Mater.

23. R. E. Stoller, J. Nucl. Mater. 276 (2000) 22-32.

24. D. J. Bacon, F. Gao, and Yu. N. Osetsky, J. Nucl. Mater. 276 (2000) 1-12.

25. R. E. Stoller and A. F. Calder, J. Nucl. Mater. 283-287 (2000) 746-752.

26. K. Nordlund and R. S. Averback, J. Nucl. Mater. 276 (2000) 194-201.

27. Yu. N. Osetsky, J. Nucl. Mater. 276 (2000) 65-77.

28. B. D. Wirth, G. R. Odette, D. Maroudas, and G. E. Lucas, J. Nucl. Mater. 276 (2000) 3340.

29. D. Rodney and G. Martin, Phys. Rev. B 61 (2000) 8714.

30. C. S. Becquart, C. Domain, A. Legris, and J. C. Van Duysen, J. Nucl. Mater. 280 (2000) 73-85.

31. A. Souidi, M. Hou, C. S. Becquart, and C. Domain, J. Nucl. Mater. 280 (2001) 179-188.

32. Yu. N. Osetsky and D. J. Bacon, Model. Simul. Mater. Sci. Eng. 11 (2003) 247. 
33. J.S. Robach, I.M. Robertson, B.D. Wirth and A. Arsenlis, Phil. Mag. 83 (2003) 995.

34. Yu. N. Osetsky, D. J. Bacon and V. Mohles, Phil. Mag. 83 (2003) 3623.

35. D. Rodney, Acta Mater. 52 (2004) 607.

36. R.O. Scattergood and D.J. Bacon, Acta Metall. 20 (1982) 1665.

37. R. P. Gupta, G. martin, S. Lanteri, P. Maugis, and M. Gyttman, Phil. Mag. A 80 (2000) 2393-2403.

38. M. Athènes, Phys. Rev. E 66 (2002) 16701:1-10 and Phys. Rev. E 66 (2002) 46705:1-14.

39. F. Soisson and G. Martin, Phys. Rev. B 62 (2000) 203-214 and; Y. Le Bouar and F. Soisson, Phys. Rev. B65 (2002) 094103-1-094103-14.

40. J.L. Bocquet, Defect and Diffusion Forum 203-205 (2002) 81.

41. J. Dalla Torre, J.L. Bocquet, N.V. Doan, E. Adam, and A. Barbu, Phil. Mag. 85 (2005) 549-558.

42. C.C. Fu, J. Dalla Torre, F. Willaime, J.L. Bocquet and A. Barbu, Nature Materials 4 (2005) 68-74.

43. M. Nastar, Defect and Diffusion Forum 194-199 (2001) 171; and in Microstructural Processes in Irradiated Materials, TMS Symposium, San Diego, March 2003; Phil. Mag. 85 (2005) 641-647.

44. F. Christien and A. Barbu, J. Nucl. Mater. 324 (2003) 90.

45. R. E. Stoller and G. R. Odette, J. Nucl. Mater. 131 (1985) 118-125.

46. R. E. Stoller and G. R. Odette, "A Composite Model of Microstructural Evolution in Austenitic Stainless Steel Under Fast Neutron Irradiation," Radiation-Induced Changes in Microstructure, 13th International Symposium, ASTM STP 955, F. A. Garner, N. H. Packan and A. S. Kumar, Editors, American Society of Testing and Materials, Philadelphia, 1987, pp. 371-392.

47. D.J. Bacon, U.F. Kocks and R.O. Scattergood, Phil. Mag. 28 (1973) 1241.

48. H. M. Zbib, V. Bulatov and T. Diaz de la Rubia, Trans. ASME 74 (2002) 78.

49. N. M. Ghoniem, S.-H. Tong, and L. Z. Sun, Phys. Rev. B 139 (2000) 913-927.

50. J. Marian, W. Cai and V. Bulatov, Nature Materials 3 (2004) 158.

51. V. Bulatov, W. Cai, J. Fier, M. Hiratani, T. Pierce, M. Tang, M. Rhee, K. Yates and T. Arsenlis, Supercomputing 2004, submitted.

52. R. L. Klueh, J. P. Shingledeckerm R. W. Swindeman, and D. T. Hoelzer, J. Nucl. Mater. 341 (2005) 103-114.

53. M. K. Miller, E. A. Kenik, K. F. Russell, L. Heatherly, D. T. Hoelzer, and P. J. Maziasz, Mat. Sci. and Engr. A353 (2003) 140-145. 
54. M.A. Sokolov, D.T. Hoelzer, M.K. Miller, and R.E. Stoller, "Fracture Toughness and Tensile Properties of Nanostructured Ferritic Steel," presented at Eleventh International Conference on Composites/Nano Engineering, Hilton-Head Island, SC, August 8-14, 2004.

55. P. Olsson, L. Malerba, and A. Almazouzi, "A First Step Towards a Multiscale Modelling of Fe-Cr Alloys,” SCK-CEN-BLG-950, Belgian Nuclear Research Center, Mol, Belgium, June, 2003.

56. E. Vincent, C. S. Becquart, and C. Domain, Nucl. Inst. and Meth. B228 (2005) 137-141.

57. C. Domain, C. S. Becquart, and, J. Foct, Phys. Rev. B69 (2004) 144112:1-16.

58. E. Vincent, C. S. Becquart, and C. Domain, "Solute Interaction with Point Defects in $\alpha \mathrm{Fe}$ Under Irradiation: A Combined ab initio and Kinetic Monte Carlo Approach," presented at 2005 TMS Symposium on Microstructural Processes in Irradiated Materials, San Francisco, February, 2005, submitted to J. Nucl. Mater.

59. M. Nastar, V. Yu. Dobretsov and G. Martin, Phil. Mag. A80 (2000) 155-184.

60. V. G. Vaks, Physics Reports 391 (2004) 157-242. 